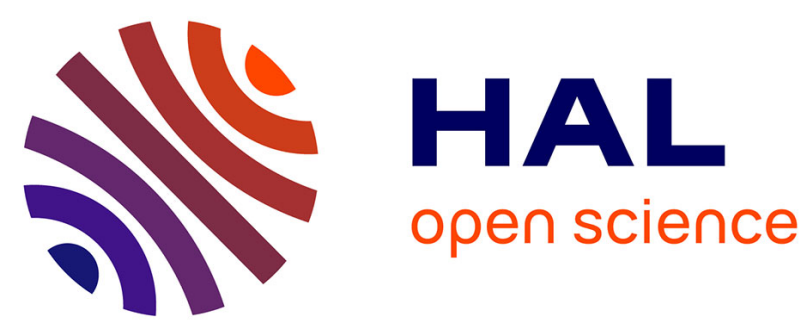

\title{
Control of Chain Alignment and Crystallization Helps Enhance Charge Conductivities and Thermoelectric Power Factors in Sequentially Doped P3HT:F 4 TCNQ Films
}

Viktoriia Untilova, Till Biskup, Laure Biniek, Vishnu Vijayakumar, Martin

Brinkmann

\section{To cite this version:}

Viktoriia Untilova, Till Biskup, Laure Biniek, Vishnu Vijayakumar, Martin Brinkmann. Control of Chain Alignment and Crystallization Helps Enhance Charge Conductivities and Thermoelectric Power Factors in Sequentially Doped P3HT:F 4 TCNQ Films. Macromolecules, 2020, 10.1021/acs.macromol.9b02389 . hal-02521023

\section{HAL Id: hal-02521023 \\ https://hal.science/hal-02521023}

Submitted on 8 Nov 2020

HAL is a multi-disciplinary open access archive for the deposit and dissemination of scientific research documents, whether they are published or not. The documents may come from teaching and research institutions in France or abroad, or from public or private research centers.
L'archive ouverte pluridisciplinaire HAL, est destinée au dépôt et à la diffusion de documents scientifiques de niveau recherche, publiés ou non, émanant des établissements d'enseignement et de recherche français ou étrangers, des laboratoires publics ou privés. 


\title{
Control of chain alignment and crystallization helps enhance charge conductivities and thermoelectric power factors in sequentially doped P3HT: $\mathrm{F}_{4}$ TCNQ films
}

\author{
Viktoriia Untilova, ${ }^{1}$ Till Biskup, ${ }^{2}$ Laure Biniek $^{1}$, Vishnu Vijayakumar ${ }^{1}$ and Martin \\ Brinkmann $^{1 *}$ \\ (1) Université de Strasbourg, CNRS, ICS UPR 22, F-67000 Strasbourg, France \\ (2) Universität des Saarlandes, Physikalische Chemie und Didaktik der Chemie, Campus B2 2, \\ 66123 Saarbrücken, Germany \\ * Corresponding author. \\ martin.brinkmann@ics-cnrs.unistra.fr
}




\begin{abstract}
Thermoelectricity has gained considerable interest in the last decade due to the advent of organic thermoelectric materials. Crystallinity and doping level crucially determine the thermoelectric figure of merit of semi-conducting polymers. Hence, detailed insight into these factors is prerequisite for developing efficient devices. Here we show that the semicrystalline structure of aligned P3HT films shows superior thermoelectric efficiencies as compared to the smectic-like phase because of both a higher in-plane orientation and a higher doping level. Conductivities up to $160 \mathrm{~S} / \mathrm{cm}$ and power factors of $56 \mu \mathrm{W} \mathrm{m}{ }^{-1} \mathrm{~K}^{-2}$ along the

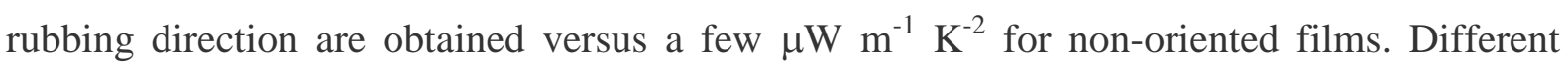
intercalation mechanisms of $\mathrm{F}_{4} \mathrm{TCNQ}$ in the layers of alkyl side chains are evidenced by electron diffraction in doped oriented films of the smectic-like and the semi-crystalline phases. We provide compelling evidence that doping of the smectic-like phase promotes ordering of P3HT backbones along the chain direction within individual $\pi$-stacks whereas for the semi-crystalline phase, dopant intercalation reorganizes the arrangement of successive $\pi$ stacks and perturbs the packing of alkyl side chains. Insight in the orientation of $\mathrm{F}_{4} \mathrm{TCNQ}^{-}$ anions in the layers of alkyl side chains of P3HT crystals was further retrieved from a detailed polarized UV-vis-NIR spectroscopic analysis. Our results demonstrate that both orientation of the polymer chains and crystallinity enhance the thermoelectric properties as well as the doping level. We anticipate that detailed control of polymer morphology in films further improves the thermoelectric figure of merit of semiconducting polymers.
\end{abstract}

Key words: organic thermoelectric, conducting polymers, structure, thin films, doping 


\section{Introduction}

The scientific community of plastic electronics witnesses in the last decade a renaissance of interest for conducting polymers and doped polymer semi-conductors used as thermoelectric materials. ${ }^{1,2}$ Besides classical conducting polymers such as PEDOT:PSS, doped polymer semi-conductors appear as interesting alternative candidates for TE applications since the processing and crystallization of the polymer semi-conductor can be decoupled from doping to ensure optimal control over both crystallinity and doping levels. It is of particular importance to master the doping process in order to tune the charge carrier densities since it determines the type of targeted application. Improved On/Off ratio and charge mobilities are obtained in Organic Field Effect Transistors (OFET) ${ }^{3,4}$ for low dopant concentrations in blends with semi-conducting and insulating polymers. Interesting thermoelectric properties of conducting polymers such as poly(3,4-ethylenedioxythiophene): poly(styrenesulfonate) (PEDOT:PSS) can be achieved by fine tuning the doping level in the thin films through sequential post-treatments with common acids and bases. ${ }^{5}$ Among other polymers, regioregular poly(3-hexylthiophene) (P3HT) doped with 2,3,5,6-tetrafluoro7,7,8,8-tetracyanoquinodimethane $\left(\mathrm{F}_{4} \mathrm{TCNQ}\right)$ is a model system to better understand the correlation between structure, charge transport and thermoelectric properties.6-19 ${ }^{6}$ The electronic process of doping in $\mathrm{P} 3 \mathrm{HT} / \mathrm{F}_{4} \mathrm{TCNQ}$ implies an integer charge transfer from the HOMO of the P3HT (approx. $-4.9 \mathrm{eV}$ ) to the LUMO of the dopant (approx. $-5.2 \mathrm{eV}$ ). ${ }^{20}$ But this redox reaction depends strongly on the proximity of dopant molecules and polymer backbone, ${ }^{10}$ hence, on the diffusion of dopant molecules and on their location in the polymer matrix. Transmission Electron Microscopy and polarized UV-Vis-NIR spectroscopy analysis of highly oriented thin films of $\mathrm{F}_{4} \mathrm{TCNQ}$-doped P3HT demonstrated that the $\mathrm{F}_{4} \mathrm{TCNQ}^{-}$anions are located in the layers of alkyl side chains within crystalline P3HT domains with the molecular long axis of $\mathrm{F}_{4} \mathrm{TCNQ}^{-}$oriented perpendicular to the polymer backbone. ${ }^{21}$ Recent 
studies on a family of poly(2,5-bis(3-alkyl-2-yl)thieno[3,2-b]thiophene) (PBTTT) with varying alkyl side chains showed that the ultimate doping level is determined by both the side chains length and packing. ${ }^{22}$ The structure of side chains (branched versus linear) is equally important in determining the ultimate doping level in thin films. ${ }^{17}$

Numerous studies on $\mathrm{P} 3 \mathrm{HT} / \mathrm{F}_{4} \mathrm{TCNQ}$ have demonstrated that the method of doping impacts the final doping level and corresponding charge conductivity. Blending P3HT with $\mathrm{F}_{4} \mathrm{TCNQ}$ in solution ${ }^{6,7}$ results in poor charge conductivities whereas sequential doping of P3HT films by spin-coating or by dipping into a solution of $\mathrm{F}_{4} \mathrm{TCNQ}^{8,9}$ leads to enhanced conductivities of up to $10 \mathrm{~S} \mathrm{~cm}^{-1}$. Even higher conductivities are obtained upon vapor phase doping. ${ }^{14,15}$ Hence a large spread of conductivities in $\mathrm{F}_{4}$ TCNQ-doped P3HT is reported in the literature depending on the doping method. Crystallinity of pristine P3HT films is also an essential factor controlling the conductivity in $\mathrm{F}_{4}$ TCNQ-doped films. ${ }^{10,11,13}$ For instance, Hynynen et al. have evidenced a clear correlation between the crystallinity of P3HT measured via the free exciton bandwidth, and the charge conductivity. ${ }^{11}$ This correlation relates to the higher charge carrier mobility as the degree of solid state order increases.

Although many studies have focused on non-oriented thin films, it can be highly advantageous to study doped oriented thin films. ${ }^{21-24}$ We have previously demonstrated that a two-step process can afford highly in-plane oriented conducting polymer layers of interest for thermoelectric applications. ${ }^{21}$ Soft doping of P3HT films rubbed at $220^{\circ} \mathrm{C}$ with a solution of $\mathrm{F}_{4}$ TCNQ in acetonitrile $(1 \mathrm{mg} / \mathrm{ml})$ in ambient leads to charge conductivities up to $22 \mathrm{~S} \mathrm{~cm}^{-1}$ and power factor of $8.5 \mu \mathrm{W} \mathrm{m}{ }^{-1} \mathrm{~K}^{-2}$. Most importantly, alignment of P3HT enhances both charge conductivity $\sigma$ and thermopower $S$ along the polymer chain direction resulting in improved TE power factors $\mathrm{PF}=\sigma \mathrm{S}^{2}{ }^{21}$ The anisotropy of the thermopower is explained in terms of a heterogeneous material model that takes into account the semi-crystalline nature of the films. ${ }^{23}$ Interestingly, adjusting the temperature applied during the rubbing process $\left(T_{R}\right)$ 
provides a handle to tune in-plane orientation, structure and crystallinity of the films. Regarding alignment, increase of $\mathrm{T}_{\mathrm{R}}$ from room temperature to $220^{\circ} \mathrm{C}$ results in a remarkable increase of the order parameter up to $0.84 .{ }^{25}$ Concerning the structure, for $50{ }^{\circ} \mathrm{C}<\mathrm{T}_{\mathrm{R}}<150$ ${ }^{\circ} \mathrm{C}$; the films consist of a small fraction of non-oriented crystalline phase and an oriented "smectic-like" phase with face-on oriented domains. In the smectic-like structure, P3HT chains show translational order along the $\pi$-stacking direction ( $\mathrm{b}_{\mathrm{P} 3 \mathrm{HT}}$ axis) but no translational order along the chain direction within stacks of backbones (the 002 reflection is absent in the diffraction pattern). In addition, the layers of alkyl side chains are highly disordered. ${ }^{25}$ When a film of $\mathrm{P} 3 \mathrm{HT}$ is rubbed above $150^{\circ} \mathrm{C}$, oriented crystallization parallel to the rubbing direction $\mathbf{R}$ sets in and leads to a well-defined semi-crystalline lamellar morphology. In the semi-crystalline phase, the packing of P3HT backbones displays well-defined translational order along both the chain and the $\pi$-stacking directions, and relatively well packed alkyl side chains. $^{26,27}$ Finally, increasing $\mathrm{T}_{\mathrm{R}}$ from $140^{\circ} \mathrm{C}$ to $220^{\circ} \mathrm{C}$ results in an increase of both crystallinity (from $25 \%$ to $60-62 \%$ ) and lamellar periodicity (that scales inversely to the undercooling).

In this contribution, we use the structural variety achieved by controlling the rubbing temperature to investigate the doping mechanism in aligned $\mathrm{P} 3 \mathrm{HT}$ films and how it impacts the resulting charge conductivity and TE properties. The fundamental questions we address are the following: How does the original structure of the P3HT films (smectic versus semicrystalline) affect the final doping level? How are the dopant molecules distributed in the structure of P3HT and to what extent are the TE properties affected? To answer these questions, we use a combination of UV-Vis-NIR absorption and electron paramagnetic resonance (EPR) spectroscopies, Transmission Electron Microscopy and Electron Diffraction, conductivity and Seebeck coefficient measurements. We uncover correlations between film structure and crystallinity and corresponding charge conductivity and TE 
properties. As a main result, the semi-crystalline structure of aligned P3HT films shows superior conductivity and TE efficiencies (power factor) as compared to the smectic-like phase. Polarized UV-vis-NIR absorption spectroscopy shows that the distinct phase behavior relates to different in-plane orientations and doping levels. A combination of electron diffraction and polarized absorption spectroscopy gives a detailed insight into the mechanism of dopant intercalation in the P3HT structure both in terms of structural modification and orientation distribution of dopant molecules in the layers of alkyl side chains. Correlations between the order parameter and the anisotropy in charge transport are analyzed. High conductivities up to $160 \mathrm{~S} / \mathrm{cm}$ and power factors of $56 \mu \mathrm{W} \mathrm{m} \mathrm{m}^{-1}$ are reached.

\section{Results and Discussion \\ 2.1 Fabrication of highly in-plane oriented conducting polymer films}

In-plane oriented P3HT thin films were prepared by mechanical rubbing at different temperatures $\mathrm{T}_{\mathrm{R}}\left(78{ }^{\circ} \mathrm{C}\right.$ to $\left.186{ }^{\circ} \mathrm{C}\right)$. The oriented P3HT films were then subjected to a soft doping by dipping the films for $10 \mathrm{~s}$ into a solution of $\mathrm{F}_{4}$ TCNQ in acetonitrile (ACN) (1 mg $\mathrm{mL}^{-1}$ ) (see Figure $1 \mathrm{a}, \mathrm{b}$ ). A particular attention has been paid to prepare the samples and perform the charge conductivity and Seebeck coefficient measurements under inert atmosphere (in our previous work, the doping process was conducted in air, resulting in lower charge conductivities). ${ }^{21}$ Polarized optical microscopy (POM) of the rubbed films shows a colour change upon doping while the films remain birefringent: the pristine orientation of the rubbed layers is not altered in the doping process (Figure ESI 1). Films rubbed at high temperature $\left(\mathrm{T}_{\mathrm{R}}>150^{\circ} \mathrm{C}\right)$ are more uniform and birefringent than those oriented at $78^{\circ} \mathrm{C}$ indicating better alignment. No rupture or discontinuity of the films normal to the rubbing are observed as demonstrated in our previous work. ${ }^{21-23}$ Rubbing temperatures above $\mathrm{T}_{\mathrm{R}}>220^{\circ} \mathrm{C}$ were not used to avoid plastic deformation of the polymer layer. 


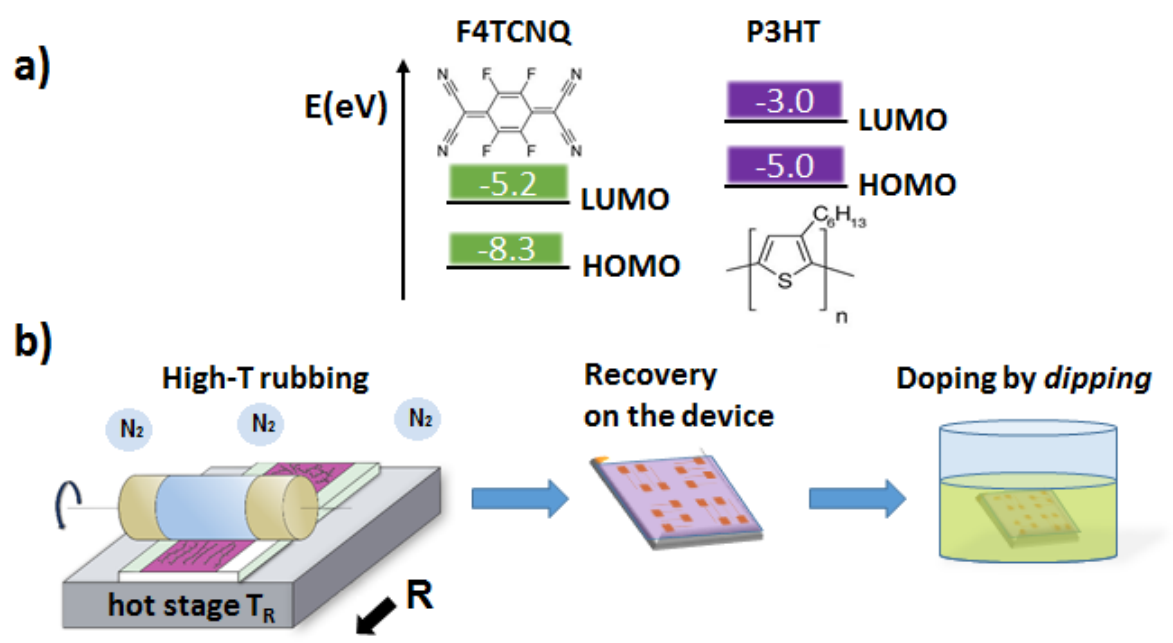

Figure 1: a) Chemical structures and schematic energy diagram of $F_{4} T C N Q$ and $P 3 H T$. b) Preparation method of in-plane oriented and conducting polymer films. First of all, chain alignment and crystallization of the P3HT films are generated by high-temperature rubbing ( $R$ is the rubbing direction). Thin oriented films are floated on distilled water and recovered on glass substrates with gold electrodes and doped by dipping into the solution of $F_{4} T C N Q$ in acetonitrile (ACN).

As doping introduces charges in the polymer, electron paramagnetic resonance (EPR) spectroscopy can be used to probe the oriented and doped P3HT films (Figure 2). ${ }^{28,29}$ This method is sensitive to the local environment of the electron spins associated with both the P3HT polaron and the $\mathrm{F}_{4} \mathrm{TCNQ}^{-}$radical anion. At X-band frequencies and fields applied here (about $9.8 \mathrm{GHz}$ microwave frequency and $0.35 \mathrm{~T}$ magnetic field strength), the contributions of these two species strongly overlap in the doped P3HT films, resulting in a featureless EPR signal whose g value of 2.0023 is close to that of the free electron. This comes to no surprise, as both, $\mathrm{P} 3 \mathrm{HT}$ polaron and $\mathrm{F}_{4} \mathrm{TCNQ}^{-}$anion, are organic radicals and the overall spectral width of at least one of them seems broader than the difference in $g$ values between the two. Despite the limited resolution of the X-band EPR spectra, evidence for both, orientation and doping is obtained for oriented and doped P3HT films. First, EPR can be used to visualize the doping process as a function of dopant concentration. As seen in Figure 2.a, the increase of doping concentration from $0.01 \mathrm{mg} / \mathrm{ml}$ to $1 \mathrm{mg} / \mathrm{ml}$ results in a clear broadening of the EPR 
signal. The broadening is attributed to increased dipolar interactions between spins, reflecting a decreased average distance between spins when the doping level augments. ${ }^{30,31}$ As an alternative, one could also consider a segregation of dopants in some parts of the films, but this contradicts the UV-vis observations indicating that only P3HT crystals are doped, not the amorphous phase (vide infra). A more direct approach to quantify the doping level would be quantitative EPR ${ }^{32}$ However, in this particular case, determining the amount of rubbed P3HT film on the substrate of each of the consecutively measured samples is far from trivial. However, we are confident that our attribution of the signal broadening to an increased dipolar interaction, hence smaller distance between $\mathrm{F}_{4} \mathrm{TCNQ}$ and P3HT, hence higher doping level, is accurate. From this, we can infer that the semi-crystalline films allow for more efficient doping as the smectic-like films (Figure 2.c), as evident from the broader EPR line of the former. ${ }^{30,31}$ Moreover, the films show a clear anisotropy of the EPR signal in the magnetic field (Figures 2.b,d). The shape (intensity and linewidth) of the signal is orientationdependent, and clearly more-for the semi-crystalline than for the smectic-like films. This gives further evidence for the highly oriented and anisotropic character of the films after doping which is further probed by polarized UV-vis-NIR spectroscopy. 
a)

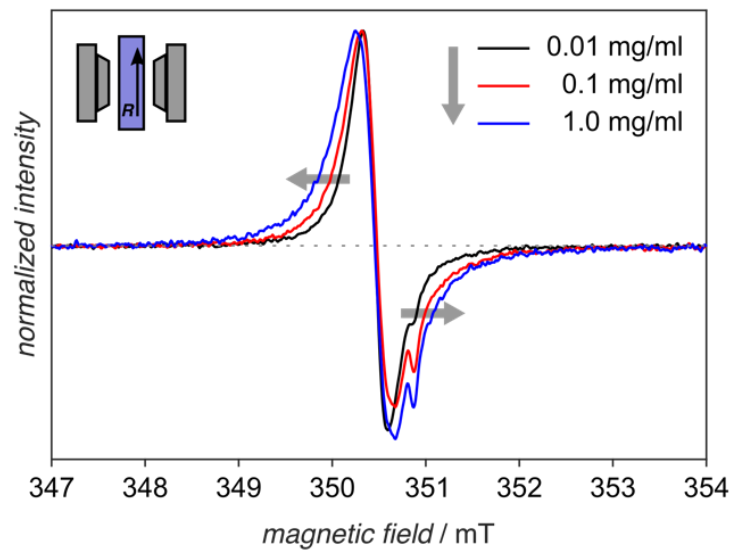

c)

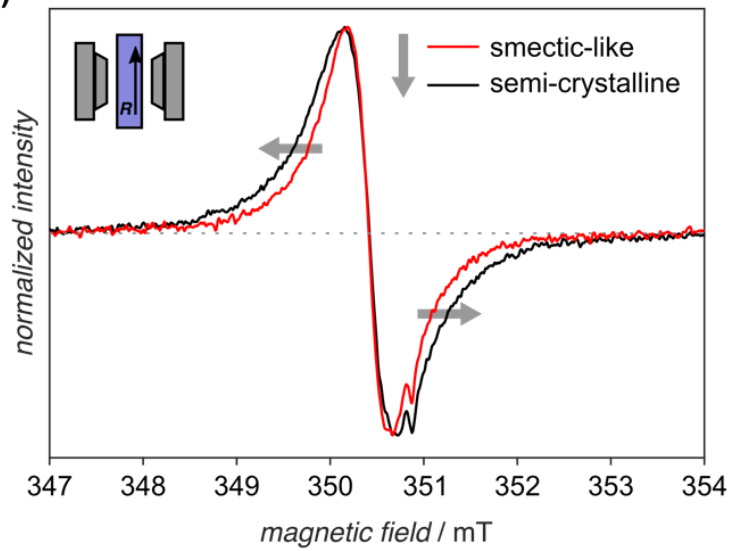

b)

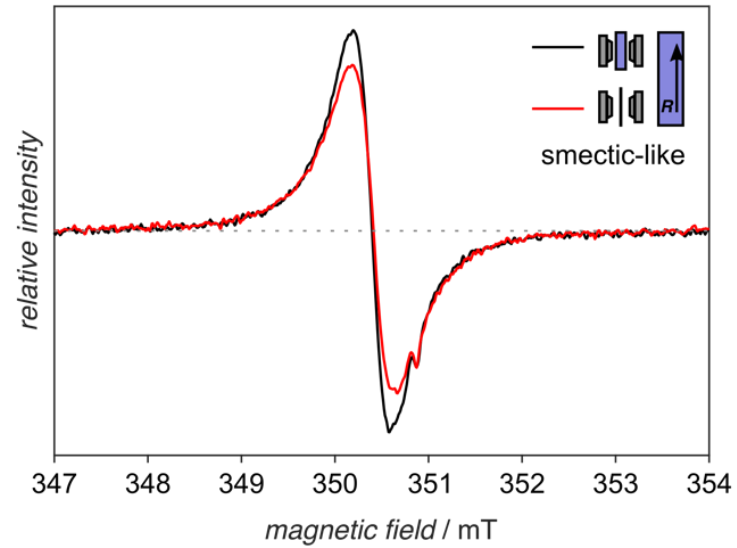

d)

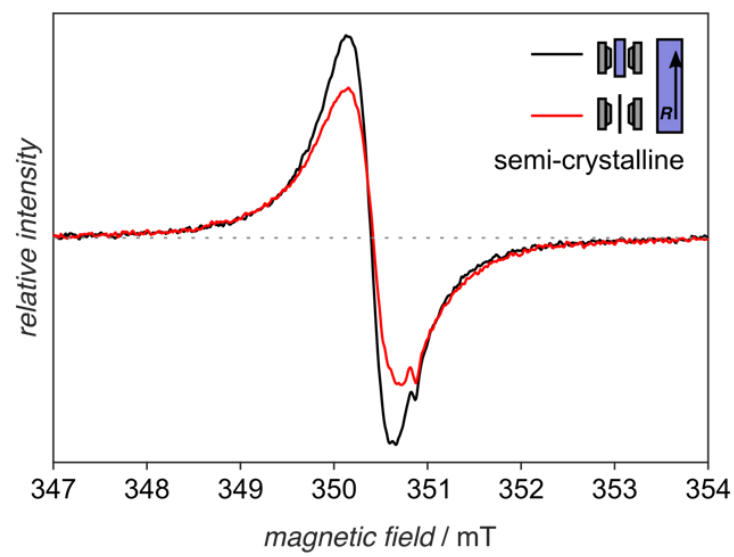

Figure 2: cW-EPR spectra obtained for P3HT doped with $F_{4} T C N Q$. (a) Spectral broadening with increasing dopant concentration. $(b, d)$ Orientation-dependence of the signal for smectic-like and semi-crystalline films. (c) Comparison of spectra for smectic-like and semi-crystalline films. The spectral broadening with increasing dopant concentration is attributed to increased dipolar interactions between the spins and can hence be directly related to an increased doping level. Therefore, the broader EPR spectra of the semi-crystalline films compared to the smectic-like films point towards an increased doping efficiency in the former. The orientation dependence of the EPR spectra, while visible for both, smectic-like and semi-crystalline films, is clearly more pronounced for the latter, as expected from their higher degree of order. The insets show the relative orientation of the pole pieces of the EPR spectrometer with respect to the thin film. In a) and c) the magnetic field lies in the film plane and is perpendicular to the rubbing direction. In b) and d) the EPR signal is recorded parallel and perpendicular to the film normal. 


\subsection{Evolution of spectroscopic features with rubbing temperature and doping}

As shown previously for $\mathrm{P} 3 \mathrm{HT}$, rubbing temperature impacts the degree of alignment and crystallinity of the films (see Figure ESI 2) ${ }^{25}$ It can therefore be used as a handle to probe the impact of structural variation on doping and thermoelectric properties. Polarized UV-Vis-NIR absorption spectroscopy evidences different levels of alignment of the rubbed P3HT films depending on the rubbing temperature $T_{R}$. The 3-D order parameter (OP) of the non-doped films increases gradually from 0 to 0.8 with increasing $T_{R}$ up to $171^{\circ} \mathrm{C}$ and levels off at 0.8 with further increase of $T_{R} \cdot{ }^{25}$ The 3-D order parameter is defined as:

$$
\mathrm{OP}=(\mathrm{DR}-1) /(\mathrm{DR}+2)
$$

With the dichroic ratio at $610 \mathrm{~nm}, \quad \mathrm{DR}=\mathrm{Abs}_{/ /} / \mathrm{Abs}_{\perp}$

Figure 3 a,b and Figure ESI 2 show the UV-vis-NIR absorption spectra for P3HT films rubbed at different $\mathrm{T}_{\mathrm{R}}$ and subsequently doped with a $1 \mathrm{mg} \mathrm{mL}^{-1}$ solution of $\mathrm{F}_{4} \mathrm{TCNQ} / \mathrm{ACN}$. Figure 3a represents the optical spectra of thin P3HT films rubbed at different $\mathrm{T}_{\mathrm{R}}$ recorded for light polarisation parallel to the rubbing direction $\mathbf{R}$ (POL // R) and normalised with respect to the thickness $(15-30 \mathrm{~nm})$. Figure $3 \mathrm{~b}$ represents the optical spectra recorded for the light polarisation perpendicular to the rubbing direction (POL $\perp$ R). The alignment is maintained after the sequential doping process (Figure ESI1). ${ }^{25}$ The spectra recorded for light polarization parallel $(\mathrm{POL} / / \mathrm{R})$ or perpendicular $(\mathrm{POL} \perp \mathrm{R})$ to the rubbing direction show different and characteristic features dominated by the doped $\mathrm{P} 3 \mathrm{HT}$ and the $\mathrm{F}_{4} \mathrm{TCNQ}^{-}$anions, respectively. For POL // R (Figure 3a), the polaronic bands P1 and P2, located at $\lambda>2500$ $\mathrm{nm}(\approx 0.5 \mathrm{eV})$ and $795 \mathrm{~nm}(1.56 \mathrm{eV})$, respectively, result from the doped and oriented P3HT backbone. The peak located at $\approx 540 \mathrm{~nm}$ corresponds to the neutral form of P3HT (labelled N). The polaronic bands are polarized along the rubbing direction, i.e. the polymer backbone. With increasing rubbing temperature, the polarization of the different species gets more defined. At low $\mathrm{T}_{\mathrm{R}}\left(<150^{\circ} \mathrm{C}\right)$, the weak anion features and weak polaronic bands are seen for 
both light polarization orientations because of the low level of alignment. On the opposite, for $\mathrm{T}_{\mathrm{R}}>150^{\circ} \mathrm{C}$, the samples are highly oriented and the different polaronic and $\mathrm{F}_{4} \mathrm{TCNQ}^{-}$bands are strongly polarized (Figure 3). For POL $\perp$ R, the UV-vis spectrum is dominated by three peaks of $\mathrm{F}_{4} \mathrm{TCNQ}^{-}$centred at 416,768 , and $875 \mathrm{~nm}$. The peak located at $480 \mathrm{~nm}$ corresponds to the amorphous fraction of P3HT. Interestingly, no polarons are observed for POL $\perp$ R at high $\mathrm{T}_{\mathrm{R}}$. This absence suggests that the amorphous interlamellar zones of P3HT are only poorly doped, as stressed in our previous study. ${ }^{21}$

For all samples, the vibronic structure of the neutral form of P3HT (N) disappears upon doping. Both, the position and the shape of the $\mathrm{N}$ component change with $\mathrm{T}_{\mathrm{R}}$. For POL // R, it red-shifts from $517 \mathrm{~nm}$ to $561 \mathrm{~nm}$ with increasing $\mathrm{T}_{\mathrm{R}}$ (Figure ESI 4.a, b). This is related to the increasing conjugation length for the P3HT films rubbed at higher $\mathrm{T}_{\mathrm{R}}{ }^{25}$ For POL $\perp \mathbf{R}$, the maximum of the absorption of neutral P3HT is slightly blue-shifted with increasing $\mathrm{T}_{\mathrm{R}}$ (from $504 \mathrm{~nm}$ to $480 \mathrm{~nm}$ ) (Figure ESI 4.c, b). This is due to the higher contribution of the amorphous part at high $T_{R}$ whereas for low $T_{R}$ both amorphous and crystalline contributions overlap.

Further insight into the doping level is obtained by following the spectroscopic variation with increasing $T_{R}$. The evolution of the absorbance of the neutral and polaronic features is of particular interest. As a general trend, the slope of the P1 band at $2500 \mathrm{~nm}$ indicates a redshift of the P1 band with increasing $\mathrm{T}_{\mathrm{R}}$. Ghosh et al. demonstrated that the redshift of the polaronic absorption correlates with a larger delocalization of the polarons in the more crystalline films. $^{33,34}$ This is consistent with the structural evolution in rubbed P3HT films, i.e. with the increase of the length of the planarized segments in P3HT crystals grown at increasing $\mathrm{T}_{\mathrm{R}}{ }^{25}$ Thus the more delocalized polaron for $\mathrm{T}_{\mathrm{R}}=186^{\circ} \mathrm{C}$ reflects the higher crystallinity of the pristine P3HT films. 
As a rule, the absorbance of $\mathrm{P} 1$ and $\mathrm{P} 2$ polaronic bands increase at the expense of the neutral form $\mathrm{N}$ with increasing $\mathrm{T}_{\mathrm{R}}$. This is clearly seen in Figure $3 \mathrm{c}$ showing the ratio $\mathrm{r}=\mathrm{Abs}$ $\mathrm{P} 1$ (at $2500 \mathrm{~nm}$ ) / $\mathrm{Abs} \mathrm{N}$ (at $550 \mathrm{~nm}$ ) as a function of $\mathrm{T}_{\mathrm{R}}$. Two temperature ranges can be defined. For $\mathrm{T}_{\mathrm{R}}<150^{\circ} \mathrm{C}, \mathrm{r} \sim 0.55$ whereas for $\mathrm{T}_{\mathrm{R}}>150^{\circ} \mathrm{C}, \mathrm{r} \sim 0.9$. This might indicate a difference in doping level for the two temperature regimes. Interestingly, the discontinuity observed around $150^{\circ} \mathrm{C}$ coincides with the transition from the smectic-like to the semicrystalline phase. ${ }^{25}$ Thus the smectic-like phase appears less doped than the semi-crystalline phase.

Let us now determine how the differences in polymer chain alignment and doping level impact the thermoelectric properties of the doped films rubbed at different temperatures. 
a) $\mathrm{POL} / / \mathbf{R}$

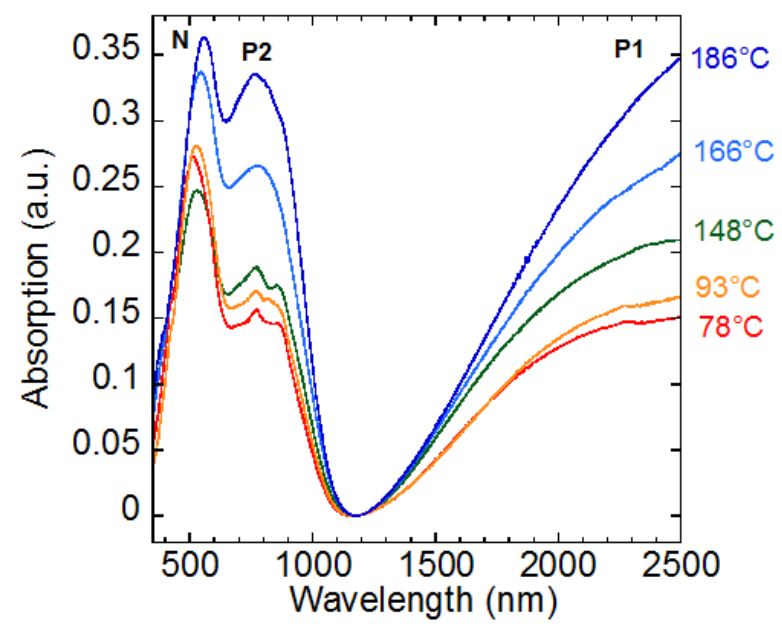

b) POL $\perp R$
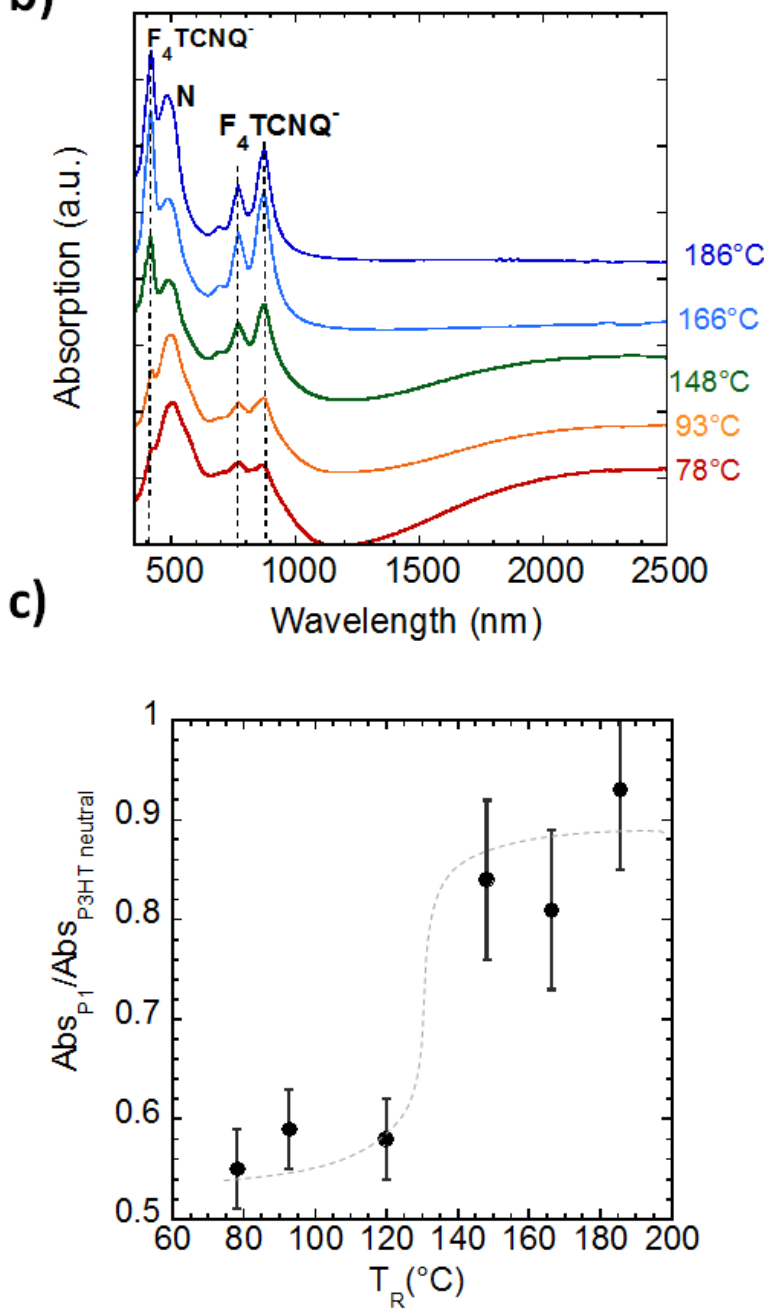

Figure 3: Evolution of the UV-Vis-NIR spectrum of highly oriented P3HT films doped with $F_{4} T C N Q(1 \mathrm{mg} / \mathrm{ml})$ as a function of the rubbing temperature $\left(T_{R}\right)$ from 78 to $186^{\circ} \mathrm{C}$. The spectra are recorded for different polarizations of the incident light with respect to the rubbing direction $(R)$. a) POL // $R$ spectra showing the polaronic bands P1 and P2 of the 
doped P3HT and the neutral P3HT (N). b) POL $\perp R$ spectra showing the absorption of neutral N P3HT (450 - $530 \mathrm{~nm})$ and the 416, 768 and $875 \mathrm{~nm}$ characteristic peaks of the $F_{4} T C N Q^{-}$anions. For clarity, the spectra for different $T_{R}$, in $P O L \perp R$, are shifted along the ordinate axis. c) Evolution of the absorbance ratio $A b s(P 1) / A b s(N)$ as a function of $T_{R}$ for doped P3HT films. The intensity of Abs (N) has been estimated by subtracting the contribution from P2 band after peak deconvolution (see the Figure ESI 7). The dashed line is a guide for the eye to highlight the transition from the smectic-like to the semi-crystalline form of P3HT.

\subsection{Dependence of thermoelectric properties on in-plane alignment and crystallinity}

In the next step, the impact of thin film structure on thermoelectric properties has been studied as a function of $T_{R}$. Charge conductivity and Seebeck coefficient were measured on thin P3HT films rubbed at $T_{R}$ from $78^{\circ} \mathrm{C}$ to $186^{\circ} \mathrm{C}$ and subsequently doped by $F_{4} T C N Q / A C N(1 \mathrm{mg} / \mathrm{mL})$. Figure 4 $\mathrm{a}, \mathrm{b}$ demonstrates the evolution of electrical conductivities $\sigma_{/ /}$and $\sigma_{\perp}$ and of the Seebeck coefficients $S_{/ /}$and $S_{\perp}$, respectively. Both $\sigma$ and $S$ are measured in parallel and in perpendicular to the rubbing direction (typical Seebeck voltage curves are shown in Figure ESI 5). The values of charge conductivity are improved along the polymer chains (delocalisation of the polarons along the polymer chains) over non-oriented films. The conductivity increases drastically above $T_{R}=140^{\circ} \mathrm{C}$. The highest value obtained for $\sigma_{\| /}$is $160 \mathrm{Scm}^{-1}$ which exceeds by one order of magnitude the value obtained for non-oriented vapor phase doped films $\left(12.7 \mathrm{Scm}^{-1}\right) .{ }^{11}$ As seen in Figure $4 a, \sigma_{\perp}$ is always below $20 \mathrm{~S} \mathrm{~cm}^{-1}$ and changes little with rubbing temperature. The charge conductivity measured perpendicular to the rubbing is close to the one obtained for the non-rubbed reference samples $(\sim 9$ $\mathrm{Scm}^{-1}$ ). It suggests that the conductivity in the non-rubbed films is limited by the contribution of the charge transport perpendicular to the polymer chains. Most interestingly, $\sigma_{/ /}$displays a discontinuity at $\sim 150^{\circ} \mathrm{C}$ that coincides again with the transition from the smectic-like to the semi-crystalline phase.

Similarly to the conductivity, the Seebeck coefficient $S_{/ /}$is enhanced along the chain direction and reaches $60 \mu \mathrm{V} \mathrm{K}{ }^{-1}$. This value does not vary much with $T_{R}$ and no discontinuity is observed around $150^{\circ} \mathrm{C}$. In contrast, $S_{\perp}$ shows a much stronger variation and decreases from $35 \mu \mathrm{V} \cdot \mathrm{K}^{-1}$ to 12 
$\mu \mathrm{V} \cdot \mathrm{K}^{-1}$ when $\mathrm{T}_{\mathrm{R}}$ approaches $160^{\circ} \mathrm{C}$. In our previous study, the anisotropy of the Seebeck coefficient was explained by a heterogeneous model taking into account the coexistence of crystalline and amorphous phases. ${ }^{23}$ In particular, it was shown that $S_{\perp}$ is determined by the Seebeck coefficient of the crystalline domains that channel the majority of charges when the temperature gradient is perpendicular to the rubbing direction. With increasing $T_{R}$ in the range $150^{\circ} \mathrm{C}-220^{\circ} \mathrm{C}$, the lamellar thickness and the average coherence length of $\mathrm{P} 3 \mathrm{HT}$ backbones in the crystalline domains tend to increase. ${ }^{21}$ In addition, the proportion of edge-on P3HT crystals inceases too. We propose that the observed variation of $S_{\perp}$ with $T_{R}$ in the semi-crystalline phase reflects these structural changes. ${ }^{21}$

Figure $4 \mathrm{c}$ shows the evolution of the power factor $\mathrm{PF}=\sigma \mathrm{S}^{2}$ as a function of $\mathrm{T}_{\mathrm{R}}$. $\mathrm{PF}_{/ /}$is

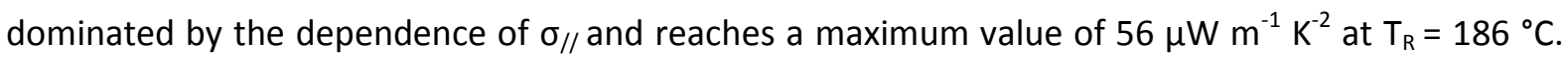
This value is higher than the $27 \mu \mathrm{W} \mathrm{m} \mathrm{m}^{-1} \mathrm{~K}^{-2}$ value reported recently for P3HT doped by vapour-phase infiltration $^{19}$ and clearly reflects the benefits of enhanced alignment and crystallinity on TE properties.

As seen from Figure $4 a, b$, explicit anisotropy of both $\sigma$ and $S$ is observed in the oriented films of P3HT. One can expect the anisotropy in conductivity to be correlated with the degree of in-plane alignment. We therefore plotted the evolution of the charge conductivity anisotropy $a_{\sigma}=\sigma_{/ /} / \sigma_{\perp}$ as a function of the order parameter extracted from the dichroic ratio of the polarons $\left(\mathrm{OP}_{\mathrm{P} 1}\right)$ as shown in Figure ESI 6. As expected, the anisotropy of charge conductivity scales with the order parameter of the polarons $\mathrm{OP}_{\mathrm{P} 1}$ which emphasizes the importance of in-plane alignment to reach the highest charge conductivities.

Having demonstrated that in-plane alignment plays an important role on charge conductivity and thermoelectric properties, the next section focuses on the impact of crystallinity on TE properties. An elegant mean to do so is to use the excitonic band width, W, as a measure of the film's crystallinity and to correlate it with the charge conductivity $\sigma_{/ /}$along the chain direction. ${ }^{11,35-37}$ Figure $4 \mathrm{~d}$ depicts the dependence of $\sigma_{/ /}$with $\mathrm{W} . \mathrm{W}$ has been extracted from the absorption spectra (POL // R) of rubbed P3HT for various $\mathrm{T}_{\mathrm{R}}$ prior to doping (see Figure ESI 4.d). The figure shows a clear 
discontinuity around $150^{\circ} \mathrm{C}$ that coincides with the structural transition from the smectic-like to the semi-crystalline phase. The $\mathrm{W}$ values of the smectic-like phase are in the 75-95 meV range whereas the semi-crystalline phase reaches $\mathrm{W}$ down to $40 \mathrm{meV}$. This result is in line with the evolution of the apparent doping level extracted from UV-Vis absorption (Figure 3c). Indeed, a similar discontinuity has been observed for the $\mathrm{P} 1 / \mathrm{N}$ absorption ratio depending on the rubbing temperature. Both results suggest that two different regimes of conductivities are effective depending on the rubbing temperature, each regime being associated to either the smectic-like or the semi-crystalline phase.

a)

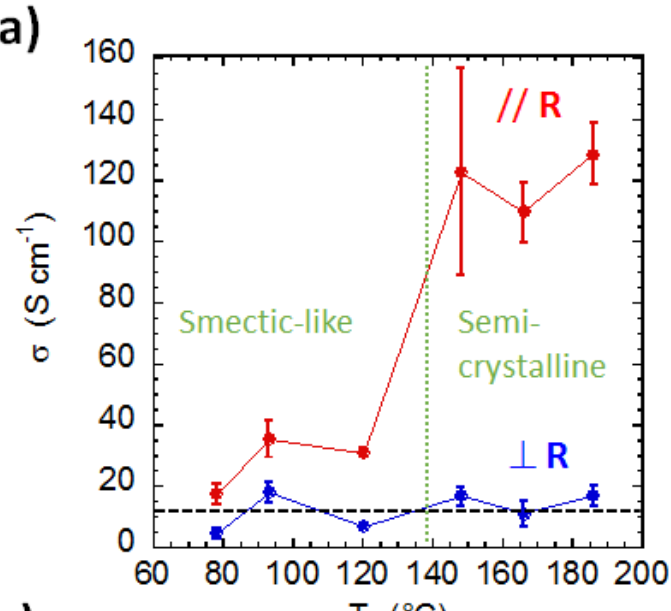

c)

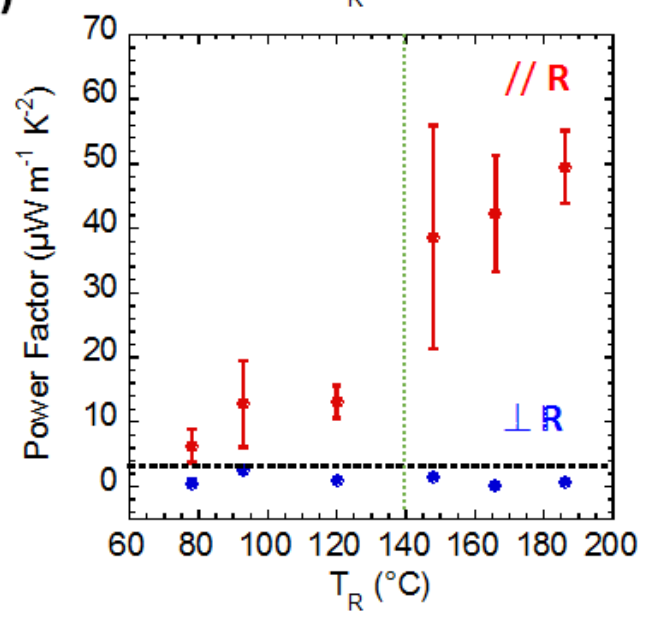

b)
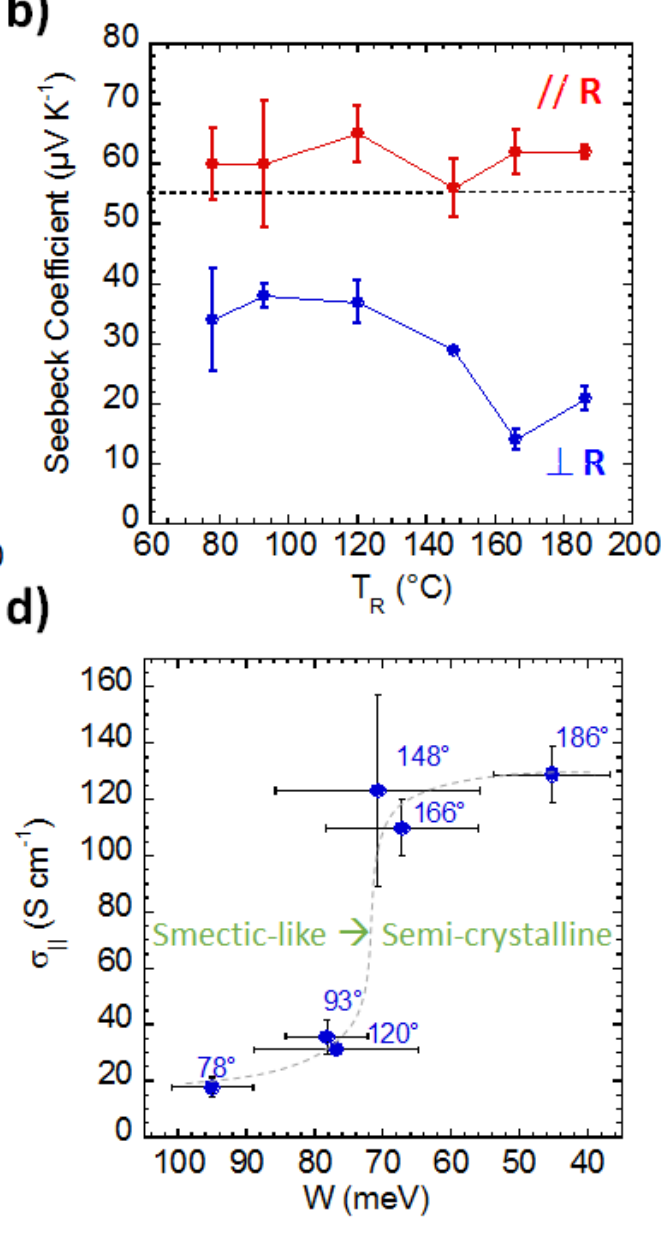

Figure 4: Evolution of the a) charge conductivity, b) Seebeck Coefficient, and c) power factor measured along $(/ / R)$ or perpendicular $(\perp R)$ to the rubbing direction for $\mathrm{P} 3 H T$ films rubbed at different temperatures and doped with $F_{4} T C N Q(1 \mathrm{mg} / \mathrm{ml})$. The black dashed lines represent the values for a non-oriented sample. The error bars are mainly related to the statistic dispersion in alignment level of samples. In a and $c$, the vertical green lines are a guide for the eye to differentiate the ranges of smectic-like and semi-crystalline phases. d) Evolution of the charge conductivity $\sigma_{/ /}$ 
measured in the direction of the polymer backbone as a function of the free exciton bandwidth $\mathrm{W}$ (see supporting information). The grey dashed line is a guide for the eye to highlight the transition between the smectic-like and the semi-crystalline phases.

The correlation between $\mathrm{W}$ and charge conductivity $\sigma_{/ /}$along the chain direction is also possibly related to the change in conjugation length induced by chain alignment upon rubbing. ${ }^{25}$ Increasing $\mathrm{T}_{\mathrm{R}}$ leads to larger stem length in the P3HT crystals and hence a higher crystallinity. The larger stem length in the crystals is consistent with the larger dichroic ratio in UV-vis absorption. As seen in Figure ESI7, this interrelation between alignment (dichroic ratio) and crystallinity (excitonic bandwidth $\mathrm{W}$ ) is clearly reflected in the apparent correlation between $\mathrm{W}$ and $\mathrm{R}$. This analysis implies that the benefits of alignment and crystallinity on TE properties are inter-related.

\subsection{Impact of doping on both smectic-like and semi-crystalline structures.}

\section{a) Structural analysis by Transmission Electron Microscopy.}

Let us now identify how the doping impacts the structure of both phases. Low dose electron diffraction was used to explore the impact of $\mathrm{F}_{4}$ TCNQ doping on the structure of P3HT thin films rubbed at different $\mathrm{T}_{\mathrm{R}}$. Figure 5 compares the ED patterns of undoped $(\mathrm{a}, \mathrm{g})$ and doped $(\mathrm{b}, \mathrm{h})$ films rubbed at $78^{\circ} \mathrm{C}(\mathrm{a}, \mathrm{b})$, and $186^{\circ} \mathrm{C}(\mathrm{g}, \mathrm{h})$ respectively. Right below the ED patterns, the corresponding section profiles along the equator $(c, i)$ and along the meridian

$(d, j)$ for doped and undoped states are shown for the films rubbed at $78^{\circ} \mathrm{C}(\mathrm{c}, \mathrm{d})$, and $186^{\circ} \mathrm{C}$ $(i, j)$. Plot profiles of the ED patterns along the equator feature the evolution of the structure along the side chains $\left(\begin{array}{lll}h & 0\end{array}\right)$ and the $\pi$-stacking ( $\left.\begin{array}{lll}0 & 2 & 0\end{array}\right)$. Meridional plot profiles help visualize the structural evolution along the polythiophene backbone $\left(\begin{array}{lll}0 & 0\end{array}\right)$. 
First, we describe the ED patterns of the non-doped films rubbed at different temperatures and identify the characteristic structural features of both smectic-like and semicrystalline phases. The P3HT films rubbed at $\mathrm{T}_{\mathrm{R}}=78^{\circ} \mathrm{C}$ are composed of a fraction of unoriented edge-on crystal (Scherrer ring 020 ) and a fraction of aligned face-on domains (equatorial $h 00, \mathrm{~h}=1-3$ ). These aligned face-on domains correspond to the smectic-like phase of P3HT as they show only equatorial $h 00$ reflections and no meridional 002 reflection along the rubbing direction. In other words, there is no well-defined translational order along the chain direction (c axis) within $\pi$-stacks of polythiophene backbones. ${ }^{25,26}$ This is illustrated in Figure 5e: (b,c) stacks show a statistical stacking disorder characterized by $\Delta \mathrm{c}_{\text {intra }}$ and disordered side chains in the smectic-like phase.

P3HT films oriented at $186^{\circ} \mathrm{C}$ have the highest degree of alignment (consistent with POM and UV-vis spectroscopy) as seen from Figures ESI 1 and ESI 3c. The films consist of both aligned edge-on and face-on P3HT crystals as indicated by the co-existence of equatorial 020 and $h 00(h=1-4)$. The 002 reflection is very sharp and intense, indicating a very high level of translational order along the chain direction c. Most characteristic of the semi-crystalline phase is the presence of sharp $h 00$ reflections $(\mathrm{h}=1-4)$ and well-defined mixed indices $h 02$ reflections, as a consequence of translational order along both ap3HT and

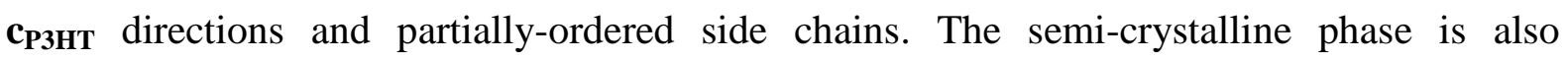
visualized by bright field transmission electron microscopy and composed of the typical periodic alternation of crystalline lamellae and amorphous interlamellar zones. ${ }^{25}$

Next, we describe the evolution of the film structure upon doping with $\mathrm{F}_{4} \mathrm{TCNQ}$. Considering the smectic-like phase, doping induces a sizable increase of the layer spacing $\mathrm{d}_{100}$ from $16.9 \AA$ to $17.9 \AA$ as well as a slight reduction in $\pi$-sacking period $\mathrm{d}_{020}$ from $3.8 \AA$ to 3.6 A. Most interestingly, some order along the monomer direction seems to be induced by doping as indicated by the appearance of the meridional 002 reflection (Figure $5 \mathrm{~b}, \mathrm{~d}$ ). In 
other words, the intercalation of $\mathrm{F}_{4} \mathrm{TCNQ}$ into the side chain layers induces a reorganisation of the polymer chains within the $(b, c)$ stacks of polythiophene backbones. Intra-stack order has thus been improved and becomes similar to that of the semi-crystalline phase but the side chains remain disordered (See schematic Figure 5f). This result is against the commonly accepted view on doping that would expect dopant intercalation to induce disorder in the pristine polymer structure at low doping concentration. ${ }^{38}$ Herein, dopant intercalation plays a role similar to solvent vapour annealing ${ }^{39}$ and allows the chains to reorganize and reach a more ordered state because the smectic-like phase is metastable and is prone to structural reorganization. It is worth noting that Chabinyc and coworkers evidenced ordering of regiorandom P3HT upon doping, indicating an effect similar to the presently one for the smecticlike phase of regioregular P3HT. ${ }^{40}$

For the semi-crystalline phase, the variation of unit cell parameters induced by doping is similar to the smectic-like phase one, albeit the variations are slightly larger along the alkyl side chains (from $16.9 \AA$ to $18.1 \AA$ ) (see Figure ESI 8). Doping induces changes in the ED pattern, especially on the second layer line: i) mixed indices $h 02$ reflections disappear and ii) the 002 reflection is streaked. Streaking in ED patterns of semi-crystalline polymers is a fingerprint of disorder. ${ }^{41}$ Streaking of the 002 is observed along the a direction, indicating that the disorder is introduced upon doping is along the $\mathbf{c}$ direction. This disordering is such that successive $(b, c)$ planes are randomly displaced along the c direction. In other words, statistical offsets $\Delta \mathrm{c}_{\text {inter }}$ are introduced between $\pi$-stacks of polythiophene backbones (see Figure $5 \ell$ ). Contrary to the smectic-like phase, $\pi$-stacking within $(b, c)$ planes is maintained whereas successive stacks experience a statistical shift $\Delta \mathrm{c}_{\text {inter }}$ upon doping. The intercalation of the $\mathrm{F}_{4} \mathrm{TCNQ}$ molecules in the side chain layers results in a disordering in the arrangement of the $(b, c)$ stacks along the side chain direction. To conclude, TEM analysis evidences two distinct cases of structural changes upon doping depending on the initial structure of the 
films. Doping improves stacking order in $(b, c)$ planes in the smectic-like phase whereas it disturbs the side chain packing and thus the relative organization of $(b, c)$ stacks in the semicrystalline phase.
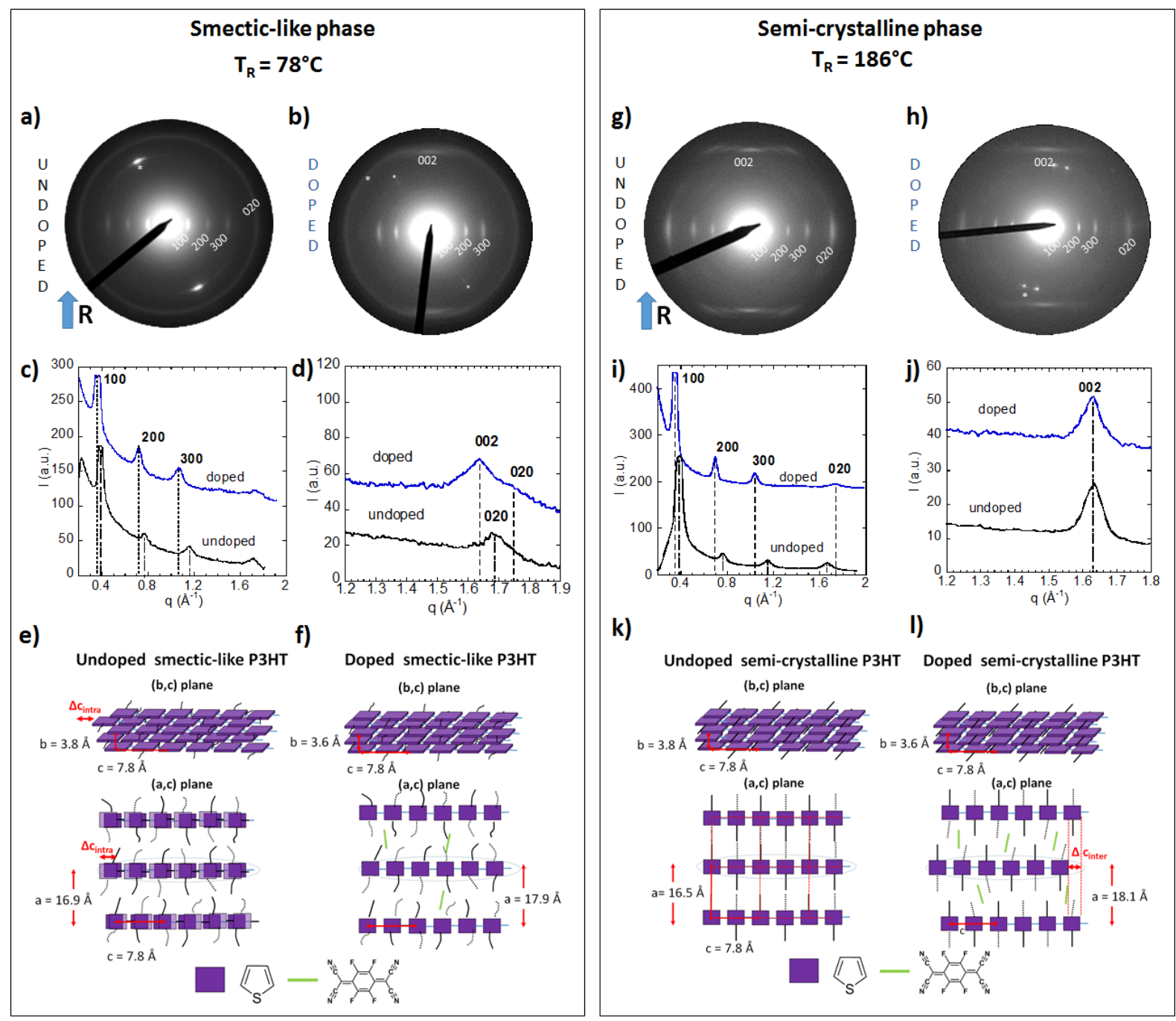

Figure 5: Electron diffraction patterns of the smectic-like $\left(T_{R}=78^{\circ} \mathrm{C}\right)$ and the semicrystalline $\left(T_{R}=186^{\circ} \mathrm{C}\right)$ films in pristine $(a, g)$ and doped $(b, h)$ forms. Equatorial $(c, i)$ and meridional ( $d$ and $j$ ) section profiles of the electron diffraction patterns. The curves in black (blue) correspond to the pristine (doped) films. For clarity, the section profiles are shifted along the ordinate axis. The asterisk seen in $a, b$ and $h$ ) indicates reflections from PTFE used for calibration. The rubbing direction $R$ is vertical (blue arrow) for all patterns. $(e, f-k, l)$ Schematic illustration of the structural changes in the packing for the smectic-like $\left(T_{R}=\right.$ $\left.78^{\circ} \mathrm{C}\right)$ and the semi-crystalline phase $\left(T_{R}=186^{\circ} \mathrm{C}\right)$ : undoped pristine structure $(e, k)$ and after doping with $F_{4} T C N Q(f, l)$. 


\section{b) Determination of in-plane orientation distribution of polarons and $\mathrm{F}_{4} \mathrm{TCNQ}^{-}$anions using UV-vis-NIR spectroscopy.}

In the previous section, the doping was shown to affect differently the structures of the smectic-like and the semi-crystalline phases. In particular, ED shows the extension of the unit cell parameters along the side chain direction but fails to determine the proportion and/or the location of the dopant molecules in the polymer matrix. In addition, the spectral features further suggest different doping levels in the two phases. Therefore, we used a detailed study of the angular dependence of the polarized UV-Vis-NIR spectra to quantify both, the angular distribution of the dopant molecules in the polymer matrix and the doping levels of both phases. The angular distribution of the P1 band reflects the in-plane distribution of the P3HT chains after rubbing and doping whereas for the $\mathrm{F}_{4} \mathrm{TCNQ}^{-}$band, it determines the in-plane angular distribution of the dopant's long axis (the transition dipole of the $\mathrm{F}_{4} \mathrm{TCNQ}^{-}$ absorption bands is oriented along the long axis of the molecule). ${ }^{42}$

Figure 6 a, b shows the evolution of the UV-Vis-NIR spectra versus the light polarization angle for smectic-like and semi-crystalline phases, respectively. The angular distribution was extracted for $\mathrm{F}_{4} \mathrm{TCNQ}^{-}$and $\mathrm{P} 1$ polarons at 873 and $2500 \mathrm{~nm}$, respectively. Since both $\mathrm{F}_{4} \mathrm{TCNQ}^{-}$and $\mathrm{P} 2$ contributions overlap in the $650-1100 \mathrm{~nm}$ range, it was necessary to deconvoluate the spectra to differentiate the two contributions and obtain meaningful absorbances for the $\mathrm{F}_{4} \mathrm{TCNQ}^{-}$anion for each angle. Figure ESI 9a shows a representative result of a multi-peak fitting of the sample rubbed at $186^{\circ} \mathrm{C}$, for a polarization angle of $70^{\circ}$, whereas Figure ESI 9c depicts the resulting $\mathrm{F}_{4} \mathrm{TCNQ}^{-}$absorbance as a function of polarization angle for the semi-crystalline films $\left(\mathrm{T}_{\mathrm{R}}=186^{\circ} \mathrm{C}\right)$ after subtraction of polymeric P2 features. 
In Figure $6 \mathrm{c}$, d, the evolution of $\mathrm{P} 1$ at $2500 \mathrm{~nm}$ (in purple) and $\mathrm{F}_{4} \mathrm{TCNQ}^{-}$at $873 \mathrm{~nm}$ (in green) bands are depicted as a function of the polarization angle, for the smectic-like and semi-crystalline phases. The curves are fitted according to equation $3::^{43,44}$

$$
A(\lambda, \theta)=A_{0}-\log \left[1-\frac{\beta}{\beta+1}(1+3 \cos 2 \theta)\right]
$$

where,

$$
\beta=\frac{1}{3} \tanh \left(\Delta A \frac{\ln 10}{2}\right)
$$

with $\mathrm{A}_{0}$, the unpolarised offset absorption contribution related to the non-aligned fraction of P3HT and $\Delta \mathrm{A}=\mathrm{A}_{\max }-\mathrm{A}_{\min }, \mathrm{A}_{\max }\left(0^{\circ}, / /\right)$ and $\mathrm{A}_{\min }\left(90^{\circ}, \perp\right)$ for $\mathrm{P} 1$ and $\mathrm{A}_{\max }\left(90^{\circ}, \perp\right)$ and $\mathrm{A}_{\min }\left(0^{\circ}, / /\right)$ for $\mathrm{F}_{4} \mathrm{TCNQ}^{-} \cdot{ }^{43,44}$

As seen in Figure $6 \mathrm{c}$, d, the angular dependence is well fitted with equation 3 for both anion and polaron contributions. In particular, the maximum of angular distribution of $\mathrm{F}_{4} \mathrm{TCNQ}^{-}$band is located at $90^{\circ}$ to the $\mathrm{P} 3 \mathrm{HT}$ backbone direction (rubbing direction). Thus, the dopant molecules are distributed around $\pm 90^{\circ}$ of the P3HT backbone direction.

For the smectic-like phase, the two curves reflect the poorly aligned structure of the film rubbed at $\mathrm{T}_{\mathrm{R}}=78^{\circ} \mathrm{C}$. Indeed, the absorbance amplitude variations of both $\mathrm{P} 1$ and of $\mathrm{F}_{4} \mathrm{TCNQ}^{-}$ are small contrary to the semi-crystalline phase. The absorption offsets $\mathrm{A}_{0}$ observed for both polarons and anions $(\sim 0.2$ and 0.05 , respectively) are related to the residual non-oriented doped P3HT crystals. For the semi-crystalline phase, the curves do not show such an offset in absorption because of the much higher alignment.

In order to quantify and compare the degree of alignment of the polaron and the anion, the dichroic ratio of both species has been used to calculate the $\mathrm{OP}$, the $3 \mathrm{D}$ order parameter according to equation 1 (see Table 1). For the smectic-like phase, both order parameters are similar and very small ( $\mathrm{OP}=0.12$ and 0.09 for $\mathrm{P} 1$ and $\mathrm{F}_{4} \mathrm{TCNQ}^{-}$, respectively), as a consequence of the large offsets $\mathrm{A}_{0}$ associated to the fraction of non-aligned P3HT in the films. In contrast, in the semi-crystalline phase, the order parameters reach much higher 
values $\left(\mathrm{OP}=0.91\right.$ and 0.62 for $\mathrm{P} 1$ and $\mathrm{F}_{4} \mathrm{TCNQ} \mathrm{Q}^{-}$, respectively). This confirms the higher alignment level of both polymer chains and dopant molecules for the films rubbed at $T_{R}>$ $150^{\circ} \mathrm{C}$. It is instructive to compare the order parameter of the polaron and the $\mathrm{F}_{4} \mathrm{TCNQ}^{-}$in the semi-crystalline phase to define to what extent their angular distributions are correlated. A perfect orientation of all dopant molecules perpendicular to the polymer backbone (inside the crystal phase) should translate into identical order parameters for the polarons and the anions. In the present case, the order parameter of the anions (0.62) is smaller than the polaron ones (0.91). The lower order parameter of the anions can have two origins: i) a fraction of $\mathrm{F}_{4} \mathrm{TCNQ}^{-}$anions is also located in the non-oriented amorphous phase, or ii) the dopants inside the layers of side chains are distributed around an average position orthogonal to the P3HT backbone.

The first origin can be ruled out because the absorption spectra recorded for POL $90^{\circ}$ (Figure $6 \mathrm{~b}$ and ESI 2c) do not show any polaronic features, suggesting that the amorphous fraction is little doped. This is also consistent with the fact that the HOMO level of P3HT in the amorphous phase is $0.1-0.2 \mathrm{eV}$ lower than that of crystalline P3HT, making it more difficult to transfer a charge between amorphous P3HT and $\mathrm{F}_{4} \mathrm{TCNQ} .{ }^{45,46}$ Therefore the nonoriented amorphous part is not responsible for the reduced order parameter.

The second origin is supported by TEM observations. The clear streaking of the 002 reflection along with the disappearance of the 102 and 202 reflections indicate a statistical rearrangement of $\pi$-stacks $\left(\Delta \mathrm{c}_{\text {inter }}\right.$ in Figure 5l) and a disordering of the packing of the side chains upon intercalation of the dopant molecules (Figure 5). The observation of disordered side chains is consistent with dopant molecules distributed close to a plane perpendicular to the P3HT backbones. The intercalation of dopants in the side chain layers appears therefore as a method to probe the side chain orientation of polymer semiconductors with respect to their backbone. If the side chains of P3HT were significantly tilted with respect to the 
backbone in the $(\mathrm{a}, \mathrm{c})$ plane, then, the maximum of the absorbance of $\mathrm{F}_{4} \mathrm{TCNQ}^{-}$anions would also be way off $90^{\circ}$, which is not observed here. Accordingly, this result supports indirectly the model of the structure of form I P3HT proposed by Kayunkid et al. stipulating that alkyl side chains are located in a plane perpendicular to the polymer backbone. ${ }^{27}$

Table 1: Main characteristics of both doped smectic-like and semi-crystalline phases

\begin{tabular}{lcc}
\hline Main characteristics of both phases & Smectic-like phase & $\begin{array}{c}\text { Semi-crystalline } \\
\text { phase }\end{array}$ \\
\hline Order parameter polaron & 0.12 & 0.91 \\
Order parameter anion & 0.09 & 0.62 \\
$\mathrm{~d}_{020} \pi$-stacking distance $(\AA)$ & 3.6 & 3.6 \\
$\mathrm{~d}_{100}$ alkyl side chains distance $(\AA)$ & 17.9 & 18.1 \\
Estimated $\mathrm{F}_{4} \mathrm{TCNQ}$ molecules per 100 thiophene & 5 & 15 \\
units & & 160 \\
Charge conductivity $\sigma_{/ /} \max \left(\mathrm{S} \mathrm{cm}^{-1}\right)$ & 35 & 56 \\
Power factor $\mathrm{PF}_{/ /} \max \left(\mu \mathrm{W} \mathrm{m}^{-1} \mathrm{~K}^{-2}\right)$ & 18 & \\
\hline
\end{tabular}

\section{c) Estimation of doping concentration.}

As shown previously, the evolution of the ratio $r=A b s$ P1 (at $2500 \mathrm{~nm}$ ) / Abs N (at 550 $\mathrm{nm}$ ) as a function of $T_{R}$ suggests that a higher doping level is obtained in the semi-crystalline phase at high $T_{R}$. To estimate this doping level in the semi-crystalline phase, differences in the angular distributions of the $\mathrm{P} 3 \mathrm{HT}$ and of the $\mathrm{F}_{4} \mathrm{TCNQ}^{-}$anion absorption bands must be taken into account. Using the extinction coefficient of $\mathrm{F}_{4} \mathrm{TCNQ}^{-}$at $870 \mathrm{~nm}\left(50000 \mathrm{M}^{-1} \mathrm{~cm}^{-1}\right.$ )$^{47}$ and the angular dependence of the pristine polymer film and the anions, it is possible to estimate a doping level defined as the number of $\mathrm{F}_{4} \mathrm{TCNQ}^{-}$anions per thiophene cycle for both structures. The obtained values (see Table 1) correspond to $5 \pm 2$ and $15 \pm 2 \%$ for the smectic-like and the semi-crystalline phase, respectively. This result is further in agreement with ESR measurements showing broader signals for the semi-crystalline phase with respect to the smectic-like phase. The upper value of the crystalline phase is consistent with a previous estimate of $17 \%$ for aligned $\mathrm{P}_{3} \mathrm{HT}^{21}$ whereas the smectic-like phase shows a 
substantially lower doping level. It is also close to the $15 \%$ doping level estimated for $\mathrm{F}_{4} \mathrm{TCNQ}^{-}$doped oriented films of $\mathrm{C}_{12}$-PBTTT. $^{22}$ This result indicates that the initial crystallinity of the P3HT films impact the doping efficiency. This is consistent with the structural study that supports different dopant intercalation mechanisms for the two phases. In the smectic-like phase, doping perturbs the stacking of P3HT backbones whereas in the semicrystalline phase, intercalation of dopants requires essentially some reorganization of the stacks along the alkyl side chains directions. Moreover, alkyl side chains are more disordered in the smectic-like phase than in the semi-crystalline phase. ${ }^{26}$ This result suggests that highly ordered alkyl side chains (non-interdigitated) favor dopant intercalation whereas highly disordered side chain layers in the smectic-like phase do not. This is consistent with recent results obtained in oriented thin films of a family of PBTTTs with side chain length varying from linear $\mathrm{C}_{8}$ to $\mathrm{C}_{18}$. The doping level was found to be lower for $\mathrm{C}_{8}$-PBTTT with disordered $\mathrm{C}_{8}$ side chains than for $\mathrm{C}_{12}$-PBTTT showing more ordered side chains. ${ }^{22}$ However; the side chains are interdigitated in $\mathrm{C}_{12}$-PBTTT which makes the diffusion of dopants more difficult than in the non-interdigitated side chains of P3HT. The ordered side chains of P3HT help orient the dopants in its unit cell and favour fast diffusion and intercalation.

The fact that the structure of the pristine films impacts the doping level in thin films is at variance with recent results by Hynynen et al. ${ }^{11}$ It was found that the doping level and the charge carrier density remain roughly constant regardless of the crystallinity of P3HT films processed in different solvents. The increase in conductivity with crystallinity was attributed to an increase in carrier mobility. In the present case, the order of the side chains seems to impact the doping level and thus the charge carrier density. A possible reason for this discrepancy could be related to the way the doping level is extracted from the absorbance of the $\mathrm{F}_{4} \mathrm{TCNQ}^{-}$anions. Indeed, rubbed P3HT films consist of a majority of face-on crystals whereas spin-coated and cast films are essentially composed of edge-on crystals. ${ }^{11,10}$ Since 
the transition dipole of the $\mathrm{F}_{4} \mathrm{TCNQ}^{-}$is along the molecular long axis, the absorbance of the $\mathrm{F}_{4} \mathrm{TCNQ}^{-}$molecules probed using polarized UV-vis-NIR spectroscopy depends on the average orientation of the dopant in the film plane. In the work of Hamidi-Sakr et al. on oriented face-on $\mathrm{P} 3 \mathrm{HT}$, the absorption of the $\mathrm{F}_{4} \mathrm{TCNQ}^{-}$anions was polarized in-plane in a direction orthogonal to the P3HT backbone, supporting the dopant intercalation in the layers of alkyl side chains and parallel to side chains. ${ }^{21}$ If the P3HT crystals are essentially edge-on, then, upon intercalation inside the layers of alkyl side chains, the long axis of the $\mathrm{F}_{4} \mathrm{TCNQ}^{-}$ molecules will be mainly distributed around the normal to the film surface. Such an orientation renders the $\mathrm{F}_{4} \mathrm{TCNQ}^{-}$invisible using in-plane UV-vis-NIR absorption measurements. Accordingly, UV-vis-NIR spectroscopy on edge-on oriented P3HT can lead to an under-estimation of the proportion of $\mathrm{F}_{4} \mathrm{TCNQ}^{-}$. This simple reasoning uncovers the intrinsic difficulty to evaluate the doping concentration from in-plane spectroscopic measurements based on $\mathrm{F}_{4} \mathrm{TCNQ}^{-}$. A more accurate mean to probe the doping efficiency might be to compare the absorbances of polaronic features. Indeed, contrary to $\mathrm{F}_{4} \mathrm{TCNQ}^{-}$, the polymer chains lie almost exclusively in the film plane, hence, the polaronic bands are polarized in-plane. From that perspective, the comparison of the smectic-like and semicrystalline phases suggests quite similar levels of doping, which is more in line with the results by Hynynen et al. ${ }^{11}$ Accordingly, the difference in charge conductivity between smectic-like and semi-crystalline phases would be more related to the difference in charge mobility due to enhanced aligned in thin films prepared at $\mathrm{T}_{\mathrm{R}}=186^{\circ} \mathrm{C}$. 
a) Smectic-like phase

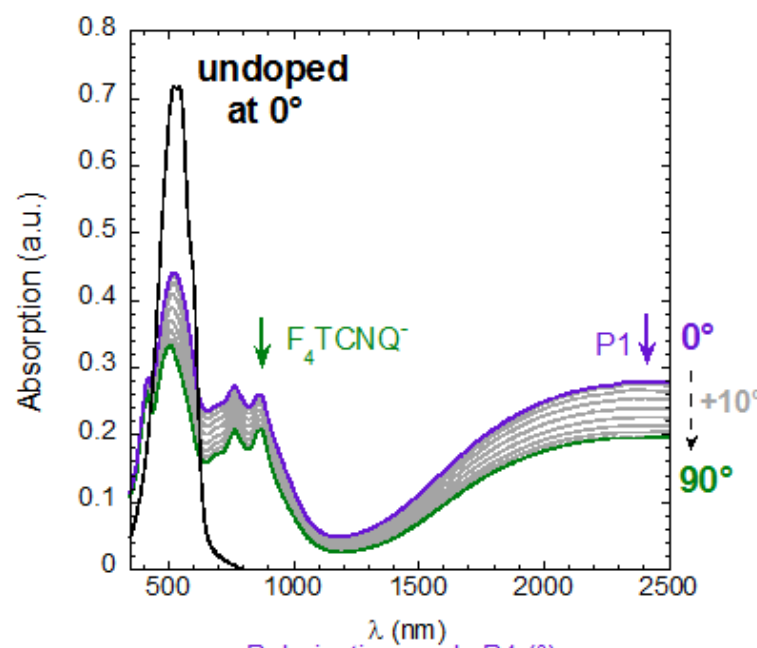

c)

Polarization angle P1 $\left(^{\circ}\right)$

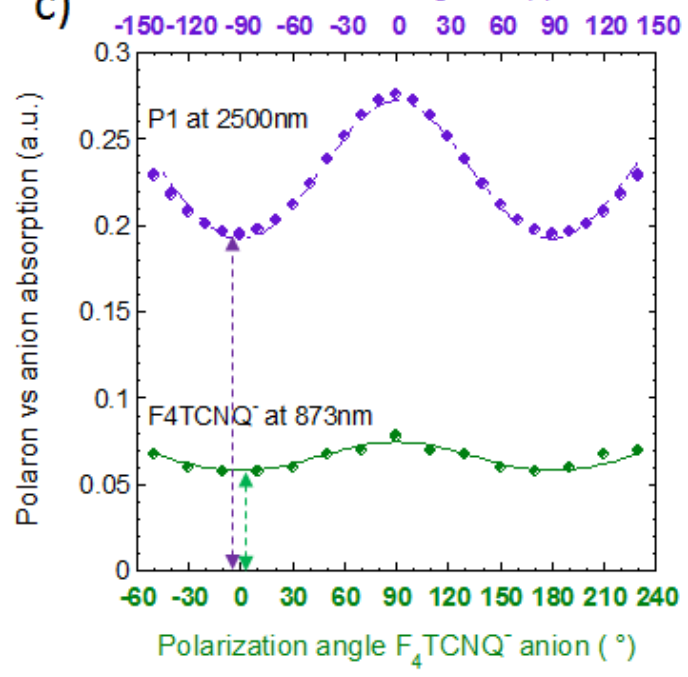

e)

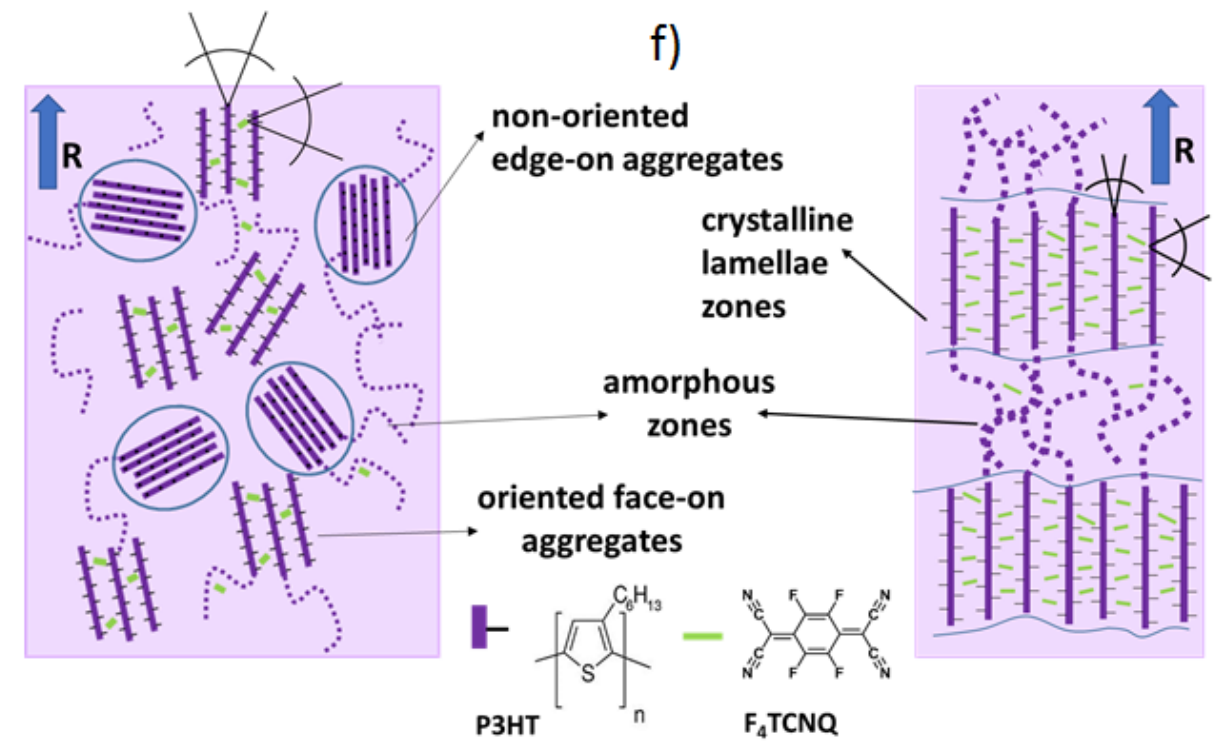

b) Semi-crystalline phase

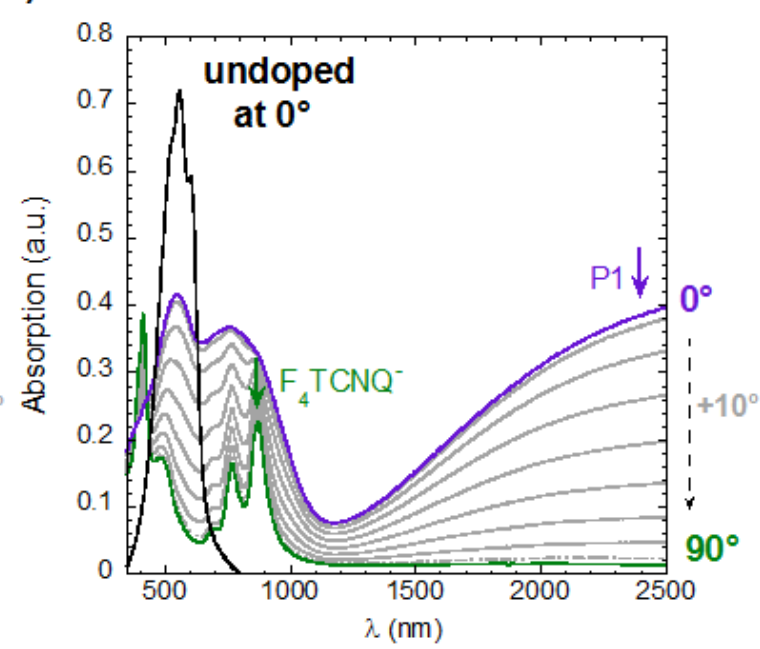

d)

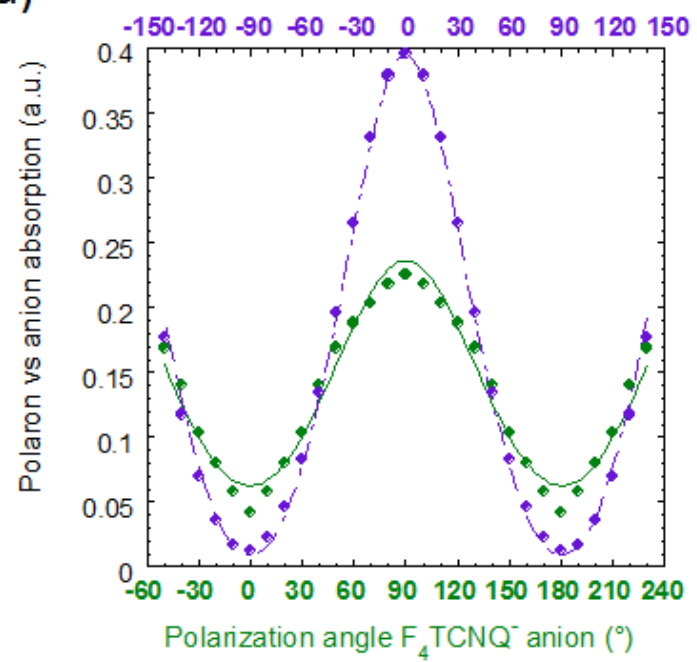

.

Figure 6: a) and b) Polarized UV-Vis-NIR absorption spectra of oriented conducting polymer films for both smectic-like and semi-crystalline phase plotted for different angles 
(every $10^{\circ}$ ) between the direction of polarization and the direction of rubbing of the film. $0^{\circ}$ corresponds to the incident light polarized parallel to the rubbing direction (POL // $R$, in violet) whereas $90^{\circ}$ corresponds to the incident light polarized perpendicular to the rubbing direction (POL $\perp R$ in green). The spectra of the rubbed undoped films at $0^{\circ}$ are also shown in black for comparison. $c$ and d) Evolution of the absorbance of the P1 polaron band at $2500 \mathrm{~nm}$ (in violet), and of the $F_{4} T C N Q^{-}$band at $873 \mathrm{~nm}$ (in green) as a function of the polarization angle for both phases, respectively. The offset in absorption represented by the dashed double arrows observed in particular for the smectic-like phase are due to the poorer alignment. e and f) Schematic representations of the structure of both doped smectic-like and semi-crystalline phases. Average orientation and location of the dopants molecules are shown in green.

\section{Conclusions}

High-temperature rubbing helps to enhance substantially the TE properties in oriented P3HT: $\mathrm{F}_{4}$ TCNQ thin films. The rubbing temperature is a precise handle to reach high crystallinity $(>55 \%$ ) and orientation (order parameter $>0.8$ ) in pristine P3HT films. Sequential doping with $\mathrm{F}_{4}$ TCNQ transforms the semi-conducting films into highly conducting polymer films with charge conductivities up to $160 \mathrm{~S} \mathrm{~cm}^{-1}$ and power factor of 56 $\mu \mathrm{W} \mathrm{m} \mathrm{m}^{-1} \mathrm{~K}^{-2}$ that lie beyond the state of the art for P3HT: $\mathrm{F}_{4} \mathrm{TCNQ}$. Furthermore, this particular and efficient tuning method of polymer chain alignment allows the fine structural analyses by TEM (in particular along the polymer chain) not accessible by conventional GIXD analyses on randomly oriented films. Polarized absorption spectroscopy gives access to the angular distribution of dopants in the polymer matrix. The combination of both techniques provides a clearer view on the doping mechanism which depends on the initial structure of the films, i.e. smectic-like vs semi-crystalline phase. In the semi-crystalline phase, well-ordered side chains help the intercalation of a larger number of dopant molecules that are oriented perpendicular to the polymer backbone. The intercalation of the $\mathrm{F}_{4} \mathrm{TCNQ}$ molecules in the side chain layers results in a disordering of both, the arrangement of 
successive $(b, c)$ polymer stacks and the packing of the side chains. In comparison, the less ordered smectic-like phase is less doped and the intercalation of $\mathrm{F}_{4}$ TCNQ into the side chain layers seems to induce a reorganisation of the polymer chains within the $(b, c)$ polymer stacks. This counter intuitive phenomenon of doping inducing order is demonstrated for the first time for regioregular P3HT. Although the semi-crystalline phase shows larger thermoelectric power factors than the smectic-like phase, it will be important to further investigate the impact of structural changes on the thermal conductivity to ascertain which of the two phases is most favorable for TE applications.

It is interesting to note that high performances reported in this study have been obtained although an important part of the film is still amorphous. Engineering of polymer crystallinity, prior to doping, could be an efficient strategy to further enhance the thermoelectric properties. Alternatively, one may also try to further preserve the pristine structure of the films, e.g. by vapor phase doping to intercalate the dopants in a less destructive manner. This reinforces the interest for new fabrication methods of highly structure-controlled conducting polymer films for thermoelectric applications.

\section{Experimental section}

Orientation and doping of thin films: regioregular P3HT was purchased from Merck $(\mathrm{Mw}=43.6 \mathrm{kDa}, \mathrm{PDI}=1.8) . \mathrm{F}_{4} \mathrm{TCNQ}$ and anhydrous solvents such as acetonitrile and orthodichlorobenzene were obtained from Sigma-Aldrich and used without purification. The films were prepared by doctor-blading a solution of P3HT in ortho-dichlorobenzene $\left(10 \mathrm{mg} \mathrm{mL}^{-1}\right)$ at $160^{\circ} \mathrm{C}$ on glass slide substrates pre-covered with a sacrificial hydrosoluble NaPSS (10 mg $\mathrm{mL}^{-1}$ aq) layer. The orientation of the films by high temperature-rubbing followed the

protocol described in previous publications. ${ }^{21,47-48}$ Oriented polymer films were prepared by using a homemade set up consisting of a translating hot plate on which the sample is fixed 
and a rotating cylinder covered with a microfiber cloth. The films were rubbed at different rubbing temperatures. To determine the film thickness after rubbing, the films were meltannealed to randomize the in-plane chain direction and the thickness was extracted from the UV-vis absorbance.

The doping was performed following the sequential doping method $^{8}$ with full sample immersion for $10 \mathrm{~s}$ in the dopant solution at a constant concentration of $1 \mathrm{mg} \mathrm{mL}^{-1}$ in anhydrous acetonitrile. Such short doping times are sufficient to reach saturation of the film doping level as shown earlier by Jacobs et al. ${ }^{8}$ No rinsing step was conducted as it may lead to dedoping of the films. Doping as well as rubbing were performed under nitrogen atmosphere.

Structural analysis by TEM. Oriented areas were identified for TEM analysis by optical microscopy (Leica DMR-X microscope). The polymer films were coated with a thin amorphous carbon film and removed from the glass substrate by floating on water and subsequent recovery on TEM copper grids. TEM was performed in bright field and diffraction modes using a CM12 Philips microscope equipped with a MVIII (Soft Imaging System) charge coupled device camera.

Calibration of the reticular distances in the ED patterns was made with an oriented polytetrafluoroethylene (PTFE) film. Beam exposure was set to a minimum using the low dose system to avoid dedoping under the electron beam that is observed when the same zone is exposed for a prolonged period of time. Dedoping is clearly manifested in the ED pattern by a change in reticular distances to those of the pristine undoped P3HT films.

Polarized UV-Vis-NIR absorption. The orientation of the polymer films was probed by UV-vis-NIR absorption (350-2500 nm) using a Varian Cary5000 spectrometer with polarized incident light and spectral resolution of $1 \mathrm{~nm}$. All spectra in Figure 2 were normalized by taking into account the thickness of each film (when fully disoriented) and 
normalizing it with respect to a reference sample. The film thickness was in the range $15-30$ $\mathrm{nm}$ (measured from the optical density after melting the films at $315^{\circ} \mathrm{C}$ for $5 \mathrm{~min}$ ).

EPR Measurements. The films for EPR measurements were prepared by floating doctor-bladed and rubbed films on quartz substrates (Ilmasil PS, QSIL GmbH) with dimensions of $3 \times 25 \mathrm{~mm}$ and a thickness of $0.2 \mathrm{~mm}$. Inside the glovebox, each sample was carefully sealed in a synthetic quartz glass tube (Ilmasil PS, QSIL GmbH) with dimensions of 3.8x3.0x240mm using Critoseal vinyl plastic. X-band EPR spectroscopy was performed at room temperature on an Elexsys 580 (Bruker Biospin $\mathrm{GmbH}$ ) spectrometer equipped with a 4119HS-W1 (Bruker) cavity. Typical acquisition parameters are: microwave frequency: 9.830 $\mathrm{GHz}$; microwave power: $150 \mathrm{~mW}$ (30 dB attenuation, $150 \mathrm{~mW}$ source power); modulation frequency: $100 \mathrm{kHz}$; modulation amplitude: $0.1 \mathrm{mT}$.

Charge conductivity and Seebeck coefficient. All devices were fabricated on glass substrates, cleaned by ultrasonication in acetone, ethanol, hellmanex and deionized water (x3 times). The cleaned substrates were dried under nitrogen and exposed to plasma prior to film deposition. Gold electrical contacts (40 nm thick) in a four-points probe geometry $(1 \mathrm{~mm}$ spacing between electrodes, $5 \mathrm{~mm}$ length) were deposited via controlled thermal evaporation through a shadow mask, at an average rate of $4-6 \AA / \mathrm{s}$. A first layer of chromium $(2.5 \mathrm{~nm}$ thick) was deposited prior the gold to promote a good adhesion on the glass substrates (evaporation rate $0.5-1 \AA / s$ ).

The geometry of deposited gold electrodes allows determining the charge transport and thermopower on a same substrate in both parallel and perpendicular direction to rubbing (see Figure S3 reference 21). Oriented films of P3HT were floated on water and carefully recovered on the device with pre-deposited gold electrodes. They were subsequently doped by dipping in the solution of $\mathrm{F}_{4} \mathrm{TCNQ} / \mathrm{ACN}(1 \mathrm{mg} / \mathrm{ml})$ for $10 \mathrm{~s}$. 
Four-point probe measurements of electrical conductivity were performed using a Keithley 4200-SCS and a Lab Assistant Semiprobe station in a Jacomex glovebox under $\mathrm{N}_{2}$ atmosphere. To derive the resistivity $\rho$ from the sheet resistance $\mathrm{R}$ measured on the device, the geometrical correction factor $\mathrm{C}$ was first determined such that $\rho=$ R.C.t where $t$ is the film thickness. To that aim, a classical four-point probe system was used on a non-oriented doped P3HT film to obtain a reference value of the resistivity. Using this value of the resistivity, the geometrical correction factor was determined for four-line electrode geometry by measuring the sheet resistance on the same sample and obtained $\mathrm{C}=1.81$, which gives $\rho=$ 1.81 R.t. The average conductivity value for a given rubbing temperature was taken as the average of two to four devices.

Thermopower measurements were conducted in nitrogen atmosphere on the same devices. The thermopower was measured using a differential temperature method whereby a temperature gradient is established across the sample along or perpendicular to the rubbing direction. The details of the experimental setup are given in reference 21 . Calibration of the Seebeck coefficient measurement was performed using a constantan wire (see ESI 10). Most importantly, the temperatures of the hot and cold gold/doped-polymer junctions are precisely defined as they lie atop the underlying Peltier elements whose temperatures are well defined and determined by an IR sensor. ${ }^{21}$ As a consequence, we do not observe a dependence of the Seebeck coefficient with the contact geometry. ${ }^{50}$

\section{Acknowledgements}

Bernard Lotz is acknowledged for careful reading of the manuscript and Matthias Pauly for fruitful discussions on angular polarization dependence. We thank Christian Blanck and Marc Schmutz for technical support in TEM. We thank Patrick Allgayer and Laurent Herrmann who contributed to the design and fabrication of the rubbing machine and Nicolas Zimmermann who prepared the pre-patterned devices. Financial supports from the ANR 
Anisotherm (ANR-17-CE05-0012) and CNRS grant PEPS Thermobody are gratefully acknowledged. Viktoriia Untilova is grateful for financial support from IRTG Softmatter / Région Grand'Est.

\section{Conflicts of interests.}

The authors declare no conflict of interest.

\section{Supporting Information}

The Supporting Information is available free of charge.

Polarized optical microscopy images of rubbed undoped and doped films; dependence of dichroic ratio on rubbing temperature, polarized UV-Vis-NIR absorption spectra of rubbed P3HT films at different rubbing temperatures and subsequently doped; evolution of the anisotropy of electrical conductivity as a function of the polaron order parameter; evolution of the $\mathrm{d}_{100}$ reticular distance as a function of $\mathrm{T}_{\mathrm{R}}$; Correlation between excitonic bandwidth and dichroic ratio; multiple peak fitting and deconvolution analysis to estimate the number of dopant molecules per thiophene units; seebeck measurements.

\section{References}

(1) Poehler, T. O. and Katz, H. E., Prospects for Polymer-based Thermoelectrics: Stat of the Art and Theoretical Analysis. Energy Environ. Sci. 2012, 8, 8110.

(2) He, M.; Qiu, F. and Lin, Z., Towards High-Performance Polymer-based Thermoelectric Materials, Energy Environ. Sci. 2013, 6, 1352. 
(3) Lu, G.; Blakesley, J.; Himmelberger, S.; Pingel, P.; Frisch, J.; Lieberwirth, I.; Salzmann, I.; Oehzelt, M.; Di Pietro, R.; Salleo, A.; Koch, N.; Neher, D., Moderate Doping Leads to High Performance of Semiconductor/Insulator Polymer Blend Transistors. Nat. Comm. 2013, 4, 1588-1596.

(4) Panidi, J.; Paterson, A. F.; Khim, D.; Fei, Z.; Han, Y.; Tsetseris, L.; Vourlias, G.; Patsalas, P. A.; Heeney, M.; Anthopoulos, T. D., Remarkable Enhancement of the Hole Mobility in Several Organic Small-Molecules, Polymers, and Small-Molecule:Polymer Blend Transistors by Simple Admixing of the Lewis Acid p-Dopant B(C6F5)3. Advanced Science 2017, 5, 1700290.

(5) Fan, Z.; Li, P.; Du, D.; Ouyang, J., Significantly Enhanced Thermoelectric Properties of PEDOT:PSS Films through Sequential Post-Treatments with Common Acids and Bases. Adv. Energy Mater. 2017, 7, 1602116.

(6) Duong, D. T.; Wang, C.; Antono, E.; Toney, M. F.; Salleo, A., The Chemical and Structural Origin of Efficient P-type Doping in P3HT. Org. Elect. 2013, 14, 1330-1336.

(7) Wang, C.; Duong, D. T.; Vandewal, K.; Rivnay, J.; Salleo, A., Optical measurement of doping efficiency in poly(3-hexylthiophene) solutions and thin films. Phys. Rev. B 2015, 91, 085205.

(8) Jacobs, I. E.; Aasen, E. W.; Oliveira, J. L.; Fonseca, T. N.; Roehling, J. D.; Li, J.; Zhang, G.; Augustine, M. P.; Mascal, M.; Moule, A. J., Comparison of Solution-Mixed and Sequentially Processed P3HT: $\mathrm{F}_{4} \mathrm{TCNQ}$ Films: Effect of Doping-Induced Aggregation on Film Morphology. J. Mater. Chem. C 2016, 4, 3454-3466.

(9) Scholes, D. T.; Hawks, S. A.; Yee, P. Y.; Wu, H.; Lindemuth, J. R.; Tolbert, S. H.; Schwartz, B. J., Overcoming Film Quality Issues for Conjugated Polymers Doped with F4TCNQ by Solution Sequential Processing: Hall Effect, Structural, and Optical Measurements. J. Phys. Chem. Lett. $2015,6,4786-4793$.

(10) Scholes, D. T.; Yee, P. Y.; Lindemuth, J.R.; Kang, H.; Onorato, J.; Ghosh, R.; Luscombe, C. K.; Spano, F. C.; Tolbert, S. H.; Schwartz, B. J., The Effects of Crystallinity on Charge Transport and the Structure of Sequentially Processed F4TCNQ-Doped Conjugated Polymer Films. Adv. Funct. Mater.2017, 27, 1702654. 
(11) Hynynen, J.; Kiefer, D.; Yu, L.; Kroon, R.; Munir, R.; Amassian, A.; Kemerink, M.; Müller, C., Enhanced Electrical Conductivity of Molecularly P-Doped Poly(3-Hexylthiophene) through Understanding the Correlation with Solid-State Order. Macromolecules 2017, 50, 8140-8148.

(12) Pingel, P.; Neher, D., Comprehensive Picture of p-Type Doping of P3HT with the Molecular Acceptor $\mathrm{F}_{4}$ TCNQ. Phys. Rev. B 2013, 87, 115209.

(13) Hynynen, J.; Kiefer, D.; Muller, C., Influence of Crystallinity on the Thermoelectric Power Factor of P3HT Vapour-Doped with $\mathrm{F}_{4}$ TCNQ. RSC Adv. 2018, 8, 1593-1599.

(14) Patel, S. N.; Glaudell, A. M.; Peterson, K. A.; Thomas, E. M.; O’Hara, K. A.; Lim, E.; Chabinyc, M. L., Morphology Controls the Thermoelectric Power Factor of a Doped Semiconducting Polymer. Science Advances 2017, 3, e1700434.

(15) Glaudell, A. M.; Cochran, J. E.; Patel, S. N.; Chabinyc, M. L., Impact of the Doping Method on Conductivity and Thermopower in Semiconducting Polythiophenes. Adv. Energy Mater. 2015, 5, 1401072.

(16) Kang, K.; Watanabe, S.; Broch, K.; Sepe, A.; Brown, A.;Nasrallah, I.; Nikolka, M.; Fei, Z.; Heeney, M.; Matsumoto, D.;Marumoto, K.; Tanaka, H.; Kuroda, S.; Sirringhaus, H. 2D coherent charge transport in highly ordered conducting polymers doped by solid state diffusion. Nat. Mater. $2016,15,896-902$.

(17) Thomas, E. M.; Davidson, E. C.; Katsumata, R.; Segalman, R. A. and Chabinyc, M. L., Branched Side Chains Govern Counterion Position and Doping Mechanism in Conjugated Polythiophenes. ACS Macro Lett. 2018, 7, 1492.

(18) Kroon, R.; Kiefer, D.; Stegerer, D.; Yu, L., Sommer, M.; Müller, C., Polar Side Chains Enhance Processability, Electrical Conductivity, and Thermal Stability of a Molecularly p-Doped Polythiophene. Adv. Mater. 2017, 29, 1700930.

(19) Lim, E.; Peterson, K. A; Su, G. M.; Chabinyc, M. L., Thermoelectric Properties of Poly(3hexylthiophene) (P3HT) Doped with 2,3,5,6-Tetrafluoro-7,7,8,8-tetracyanoquinodimethane (F4TCNQ) by Vapor-Phase Infiltration. Chem. Mater. 2018, 30, 998-1010. 
(20) Gao, W.; Kahn A., Controlled p-doping of the hole-transport molecular material N, $\mathrm{N}^{\prime}$-diphenyl$\mathrm{N}, \mathrm{N}^{\prime}$-bis(1-naphthyl)-1,1'-biphenyl-4,4'-diamine with tetrafluorotetracyanoquinodimethane. J. Appl. Phys. 2003, 94, 359.

(21) Hamidi-Sakr, A.; Biniek, L.; Bantignies, J.-L.; Maurin, D.; Herrmann, L.; Leclerc, N.; Lévêque, P.; Vijayakumar, V.; Zimmermann, N.; Brinkmann, M., A Versatile Method to Fabricate Highly InPlane Aligned Conducting Polymer Films with Anisotropic Charge Transport and Thermoelectric Properties: The Key Role of Alkyl Side Chain Layers on the Doping Mechanism. Adv. Funct. Mater. 2017, 27, 1700173.

(22) Vijayakumar, V.; Zaborova, E.; Biniek, L.; Zeng, H.; Herrmann, L.; Carvalho, A.; Boyron, O.; Leclerc, N. and Brinkmann, M., Effect of alkyl side chain length on doping kinetics, thermopower and charge transport properties in highly oriented $\mathrm{F}_{4} \mathrm{TCNQ}$ doped PBTTT films, ACS Appl. Mater. Interfaces 2019, 11, 4942.

(23) Vijayakumar, V.; Zhong, Y.; Untilova, V.; Bahri, M.; Herrmann, L.; Biniek, L., Leclerc N.; Brinkmann, M., Bringing conducting polymers to high order: towards conductivities beyond 105 $\mathrm{S} / \mathrm{cm}$ and thermoelectric power factors of $2 \mathrm{~mW} \mathrm{~m}^{-1} \mathrm{~K}^{-2}$. Adv. En. Mater. 2019, 1900266.

(24) Yasuda, T., Anisotropic carrier transport properties of stretch-oriented $\pi$ - conjugated polymers in organic field-effect transistors. Phys. Status Solidi C 8, 2011, 2, 604-606.

(25) Hamidi-Sakr, A.; Biniek, L.; Fall, S.; Brinkmann, M., Precise Control of Lamellar Thickness in Highly Oriented Regioregular Poly(3-Hexylthiophene) Thin Films Prepared by HighTemperature Rubbing: Correlations with Optical Properties and Charge Transport. Adv. Funct. Mater. 2016, 26, 408-420.

(26) Hartmann, L.; Tremel, K.; Uttiya, S.; Crossland, E.; Ludwigs, S.; Kayunkid, N.; Vergnat, C. Brinkmann, M., 2D versus 3D Crystalline Order in Thin Films of Regioregular Poly(3hexylthiophene) Oriented by Mechanical Rubbing and Epitaxy. Adv. Funct. Mat. 2011, 21, 4047.

(27) Kayunkid, N.; Uttiya, S. Brinkmann, M., Structural Model of Regioregular Poly(3hexylthiophene) Obtained by Electron Diffraction Analysis. Macromolecules 2010, 43, 4961. 
(28) Atherton, N. M. Electron Spin Resonance: Theory and Applications (Ellis Horwood Ltd., Chichester, UK, 1973).

(29) Weil, J. A. \& J. R. Bolton. Electron Paramagnetic Resonance 2nd edition (J. Wiley \& Sons, Inc., Hoboken, NJ, USA, 2007).

(30) Gao, J.; Stein, B. W. ; Thomas, A. K. ; Garcia ; J. A., Yang, J. ; Kirk M. L., Grey J. K., Enhanced Charge Transfer Doping Efficiency in J-Aggregate Poly(3-hexylthiophene) Nanofibers. J. Phys. Chem. C 2015, 119, 16396-16402.

(31) Gao, J.; Niles, E.T. ; Grey J.K., Aggregates Promote Efficient Charge Transfer Doping of Poly(3hexylthiophene). J. Phys. Chem. Lett. 2013, 4, 2953-2957.

(32) Eaton, G.R., S. S. Eaton, D. P. Barr \& R. T. Weber. Quantitative EPR (Springer, Wien, 2010).

(33) Ghosh, R.; Pochas, C. M.; Spano, F. C., Polaron Delocalization in Conjugated Polymer Films. J. Phys. Chem. C 2016, 120, 11394--11406.

(34) Ghosh, R.; Chew, A. R.; Onorato, J.; Pakhnyuk, V.; Luscombe, C. K.; Salleo, A.; Spano, F. C., Spectral Signatures and Spatial Coherence of Bound and Unbound Polarons in P3HT Films: Theory Versus Experiment. J. Phys. Chem. C 2018, 122, 18048--18060.

(35) Clark, J.; Chang, J.-F.; Spano, F. C.; Friend, R. H.; Silva, C., Determining exciton bandwidth and film microstructure in polythiophene films using linear absorption spectroscopy. Appl. Phys. Lett. 2009, 94, 163306.

(36) Spano, F. C. Modeling disorder in polymer aggregates: The optical spectroscopy of regioregular poly(3-hexylthiophene) thin films. J. Chem. Phys. 2005, 122, 234701.

(37) Spano, F. C. Absorption in regio-regular poly(3-hexyl)thiophene thin films: Fermi resonances, interband coupling and disorder. Chem. Phys. 2006, 325, 22-35.

(38) Chew, A.R.; Salleo, A., Spectroscopic studies of dopant-induced conformational changes in poly (3-hexylthiophene) thin films. MRS Communications 2017, 7, 728-734.

(39) Schulz G. L.; Ludwigs, S., Controlled Crystallization of Conjugated Polymer Films from Solution and Solvent Vapor for Polymer Electronics, Adv. Funct. Mater. 2017, 27, 1603083. 
(40) Lim, E.; Glaudell, A. M.; Miller, R. and Chabinyc, M. L. The role of Ordering on the Thermoelectric Properties of Blends of Regioregular and Regiorandom Poly(3-hexylthiophene). Adv. Elect. Materials 2019, 1800915.

(41) Lovinger, A. J.; Lotz, B.; Davis, D. D.; Padden Jr, F. J., Structure and Defects in Fully Syndiotactic Polypropylene. Macromolecules 1993, 26, 3494-3503.

(42) Haller, I.; Kaufman F. B., Spectra of tetracyanoquinodimethane monovalent anion: vibrational structure and polarization of electronic transitions. J. Am. Chem. Soc. 1976, 98, 1464.

(43) Sata, R.; Suzuki, H.; Ueno, N.; Morisawa, Y.; Hatanaka, M.; Wakabayashia, T., UV-polarizing linear polyyne molecules aligned in PVA. J. Chem. Phys. 2019, 32, 175.

(44) B. Bahadur Ed., Liquid Crystals-Applications and Uses, Vol. 3, World Scientific, 101 (1992)

(45) Jiang, X. M.; Österbacka, R.; Korovyanko, O.; An, C. P.; Horovitz, B.; Janssen, R. A. J.; Vardeny, Z. V. Spectroscopic Studies of Photoexcitations in Regioregular and Regiorandom Polythiophene Films. Advanced Functional Materials 2002, 12 (9), 587-597

(46) Tsoi, W. C.; Spencer, S. J.; Yang, L.; Ballantyne, A. M.; Nicholson, P. G.; Turnbull, A.; Shard, A. G.; Murphy, C. E.; Bradley, D. D. C.; Nelson, J.; et al. Effect of Crystallization on the Electronic Energy Levels and Thin Film Morphology of P3HT:PCBM Blends. Macromolecules 2011, 44 (8), 29442952.

(47) Dixon, D. A.; Calabrese, J. C.; Miller J. S., Crystal and Molecular Structure of the 2: 1 ChargeTransfer Salt of Decamethylferrocene and Perfluoro-7,7,8,8-tetracyano-p-quinodlmethane: $\left[\left[\mathrm{Fe}(\mathrm{C} 5 \mathrm{Me} 5)_{2}\right]^{+}\right]_{2}\left[\mathrm{TCNQF}_{4}\right]^{2-}$. The Electronic Structure of $\left[\mathrm{TCNQF}_{4}\right]_{n}(\mathrm{n}=0,1-$, 2-). J. Phys. Chem. $198993,2284-2291$.

(48) Biniek, L.; Leclerc, N.; Heiser, T.; Bechara, R.; Brinkmann, M., Large scale alignment and charge transport anisotropy of $\mathrm{pBTTT}$ films oriented by high temperature rubbing. Macromolecules 2013, 46, 4014-4023.

(49) Biniek, L.; Pouget, S.; Djurado, D.; Gonthier, E. ; Tremel, K.; Kayunkid, N.; Zaborova, E.; CrespoMonteiro, N.; Boyron, O.; Leclerc, N.; Ludwigs, S.; Brinkmann, M., High-Temperature Rubbing: A 
Versatile Method to Align $\pi$-Conjugated Polymers without Alignment Substrate Macromolecules $2014,47,3871$.

(50) Reenen, S. van; Kemerink, M. Correcting for Contact Geometry in Seebeck Coefficient Measurements of Thin Film Devices. Organic Electronics 2014, 15 (10), 2250-2255.

\section{Table of Contents}

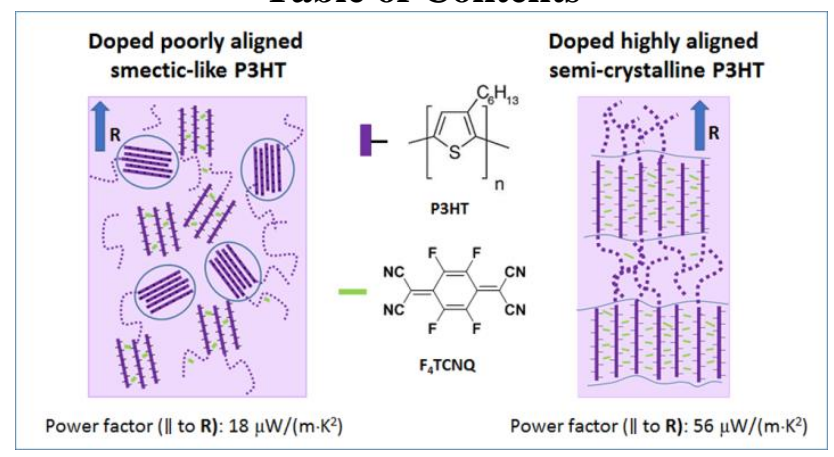

